\title{
Baroclinic flow and the Lorenz-84 model
}

\author{
Lennaert van Veen*
}

October 20, 2018

\begin{abstract}
The bifurcation diagram of a truncation to six degrees of freedom of the equations for quasi-geostrophic, baroclinic flow is investigated. Period doubling cascades and Shil'nikov bifurcations lead to chaos in this model. The low dimension of the chaotic attractor suggests the possibility to reduce the model to three degrees of freedom. In a physically comprehensible limit of the parameters this reduction is done explicitly. The bifurcation diagram of the reduced model in this limit is compared to the diagram of the six degrees of freedom model and agrees well. A numerical implementation of the graph transform is used to approximate the three dimensional invariant manifold away from the limit case. If the six dimensional model is reduced to a linearisation of the invariant manifold about the Hadley state, the Lorenz- 84 model is found. Its parameters can then be calculated from the physical parameters of the quasi-geostrophic model. Bifurcation diagrams at physical and traditional parameter values are compared and routes to chaos in the Lorenz- 84 model are described.
\end{abstract}

\section{Introduction}

The equations for atmospheric flow form one of the most intensely studied dynamical systems of the last century. Both their practical importance and their mathematical richness have attracted much attention. The atmospheric equations are studied in various forms, depending on the physical domain, and the time and length scales of interest. The starting point of the analysis in this paper is a model which describes midlatitude atmospheric flow on a synoptic scale, a few thousand kilometers in space and a week or so in time. A phenomenological description of the typical flow patterns in this range can be found in Peixoto and Oort [1992], chapter 7. The model consists of the filtered equations, derived from the basic atmospheric equations under the assumption of quasi-geostrophic (QG) and hydrostatic balance (see Holton [1992]).

In the absence of dissipative processes and forcing through solar heating, the filtered equations are energy preserving. The dominant dissipative terms are friction at the earths surface, internal friction and Newtonian cooling. The solar heating induces a strong temperature gradient in the meridional direction. Additional temperature gradients in the zonal direction can be induced by, e.g. land-sea contrast. The response to this forcing is a strong westerly circulation, called the jet stream. This circulation can become dynamically unstable so that traveling waves develop.

The jet stream pattern is nearly equivalent barotropic, which means that its height dependence can be represented by multiplication by a scale function. In other words, at each surface of constant height, the velocity field has the same shape, but may have a different amplitude. In contrast, the traveling waves can be baroclinic, which means that their phase depends on the vertical coordinate. Typically, they exhibit a westward tilt with height. Theoretical studies of the filtered equations indicate, that the baroclinicity of these traveling waves changes in the course of their life cycle

${ }^{*}$ Mathematical Institute, University of Utrecht, PO Box 80.010, 3508 TA Utrecht, the Netherlands 
[Frisius, 1998]. In the growing, strongly baroclinic, phase, they extract energy from the jet stream. In the decaying phase, they can become equivalent barotropic and transfer energy back into the jet stream.

The focus of this study is on the interaction of the jet stream and the baroclinic waves and its representation in a low order model. With the aid of discretisation in the vertical and Galerkin truncation in the horizontal coordinates, we approximate the filtered equations by a finite number of ordinary differential equations (ODE's). The discretisation can be done without violating the energy preserving nature of the filtered equations, as described in Lorenz [1960]. The number of layers is fixed to two, the minimal number necessary to describe baroclinic waves.

The two layer model is considered on an $f$-plane, a rectangular domain on which the Coriolis force is taken to be constant. The partial differential equations of the two layer model are then projected onto Fourier modes. In each layer, we use one zonally symmetric pattern, representing the jet stream, and two patterns which combine to represent a traveling wave. Thus, the ODE model has six degrees of freedom. The solar forcing is represented by constant terms, which are used as bifurcation parameters.

The bifurcation diagram is organised by its codimension two points, namely fold-Hopf, 2:1 resonance and a neutral saddle-focus on a homoclinic bifurcation line. Two routes to chaos are readily identified: period doubling cascades and a Shil'nikov type bifurcation. An inspection of the spectra of equilibria and periodic orbits found, and the calculation of the Kaplan-Yorke dimension of the chaotic attractor, leads to the conjecture, that there is a three dimensional, globally attracting, invariant manifold in the phase space of the six dimensional model.

In order to calculate a first approximation of this invariant manifold, a small parameter is introduced into the equations. In the limit where this parameter tends to zero, an analytic expression is obtained. This limit has a clear physical interpretation in terms of the energy transfer between the jet stream pattern and the traveling waves. Numerical evidence for the persistence of the invariant manifold away from this limit is obtained by the use of techniques introduced by Broer et al. [1997] and Foias et al. [1988].

Reducing the six dimensional model to the invariant manifold, in the limit where the small parameter tends to zero, a three dimensional model is obtained. Its bifurcation diagram is compared to the corresponding diagram of the six dimensional model in order to see if the qualitative dynamics is retained. This comparison is convincing. Particularly, the codimension two points are still present.

If the six dimensional model is reduced to a linearisation of the invariant manifold about the Hadley state, the Lorenz-84 model emerges. This model was introduced by Lorenz [1984] as the simplest model capable of representing the basic features of midlatitude, synoptic flow. To the author's knowledge, no derivation of the Lorenz-84 model from atmospheric flow equations has been presented before. A rather ad hoc link was established by Wiin-Nielsen [1992], but in his work the reduction to three degrees of freedom is not based on physical or mathematical arguments. The link established here enables us to calculate the parameters in the Lorenz- 84 model from the physical parameters in the filtered equations. As it turns out, one of the parameters comes out significantly different from its traditional, yet unmotivated, value. A continuation in this parameter relates the bifurcation diagram found at the traditional parameter value, presented in Shilnikov et al. [1995], to the one found at the physical value. The latter still bears resemblance to the bifurcation diagram of the six dimensional model, but the neutral saddle-focus transition is no longer there. Hence the route to chaos through a Shil'nikov type bifurcation is absent. It is shown, that chaos through period doubling cascades, the Ruelle-Takens scenario and intermittency does occur in the Lorenz- 84 model.

The derivation presented here is not unlike the derivation of the Lorenz-63 model from the fluid dynamical equations governing Rayleigh-Bénard convection Saltzman, 1962: Lorenz, 1963]. There too, the Galerkin truncation calculated has six degrees of freedom and can be reduced to a three 
dimensional invariant manifold. In that case, however, the invariant manifold is linear.

Galerkin truncations of the filtered equations have been studied on various domains and at various truncation numbers, see De Swart [1988] and references therein. One lesson to be learned from these studies, is that severe truncations, such as the one studied here, can only be regarded as qualitative models. A quantitative comparison to solutions of the filtered equations may be sensible at a truncation number in the order of a hundred or higher, depending on the basis functions used (see, e.g., Achatz and Branstator [1999 and Itoh and Kimoto [1996]). Low order models, however, allow us to isolate a physical process, such as the interaction between the jet stream and baroclinic waves, and represent it in a simple way. Because of this conceptual simplicity, and the fact that they are easy to integrate numerically, low dimensional truncations are widely used for testing and illustrating new ideas in dynamical systems theory, meteorology and climatology. The Lorenz84 model for instance, has been used to investigate low-frequency atmospheric variability Pielke and Zeng, 1994, measures of predictability González-Miranda, 1997 and time scale interaction in the climate system Roebber, 1995; van Veen et al., 2001]. The link with the filtered equations, presented here, validates the use of the Lorenz- 84 model in these contexts, though with different parameter values.

\section{The QG two layer model}

As a starting point for the calculations we take the quasi-geostrophic two layer model, described by Lorenz [1960]. Alternative derivations of this model can be found e.g. in Holton [1992], chapter 8, or the review article by De Swart [1988]. In Lorenz' article the stress is on the energy conserving nature of the nonlinear interaction terms, in De Swarts review article strong scaling arguments are provided.

\subsection{Setup of the model}

In the quasi-geostrophic approximation, the dry atmosphere is described by the velocity field, $\mathbf{v}$, and the temperature field, $T$. It is convenient to use the streamfunction, $\Psi$, the velocity potential, $\chi$, and the potential temperature, $\Theta$, as variables. As a vertical coordinate we use pressure instead of height. The velocity and the temperature can then be expressed as

$$
\begin{array}{rlrl}
\mathbf{v}_{r} & =\mathbf{k} \times \nabla \Psi & \mathbf{v}_{d} & =\nabla \chi \\
\mathbf{v} & =\mathbf{v}_{r}+\mathbf{v}_{d}+\omega \mathbf{k} & T & =\Theta\left(\frac{p}{p_{s}}\right)^{\frac{c_{p}-c_{v}}{c_{p}}}
\end{array}
$$

Here $\mathbf{v}_{r}$ and $\mathbf{v}_{d}$ are the divergence free and the irrotational part of the horizontal velocity, $p$ is pressure, $p_{s}$ is surface pressure, $\omega=\mathrm{d} p / \mathrm{d} t$ is the vertical velocity, $\mathbf{k}$ is the vertical unit vector and $c_{v}$ and $c_{p}$ are the specific heat of dry air at constant volume and pressure, respectively.

At the earth's surface, the lower boundary, we impose that $p=p_{s}$ is constant and $\omega=0$. At the upper boundary we have $p=0$ and $\omega=0$. Discretisation of the vertical in layers means that we replace each function of three spatial variables by a number of functions of longitude and latitude only. In the simplest case we take two layers, the minimal number necessary to describe baroclinic waves.

The two layers are bounded by three isobaric surfaces at $p_{0}=p_{s}, p_{2}=p_{s} / 2$ and $p_{4}=0$ (see figure (1). Vertical derivatives are replaced by linear interpolations, e.g. in the continuity equation:

$$
\nabla^{2} \chi+\frac{\partial \omega}{\partial p}=0 \rightarrow\left\{\begin{array}{c}
\nabla^{2} \chi_{3}+\omega\left(p_{2}\right)=0 \\
\nabla^{2} \chi_{1}-\omega\left(p_{2}\right)=0
\end{array} \Rightarrow \nabla^{2}\left(\chi_{1}+\chi_{3}\right)=0\right.
$$




$$
\mathrm{p}_{4}=0
$$

$$
\begin{array}{ll}
\text { Layer } 2 & \mathrm{p}_{3}=\frac{1}{4} \mathrm{p}_{\mathrm{s}} \\
\Psi_{3}, \Theta_{3} & \mathrm{p}_{2}=\frac{1}{2} \mathrm{p}_{\mathrm{s}}
\end{array}
$$

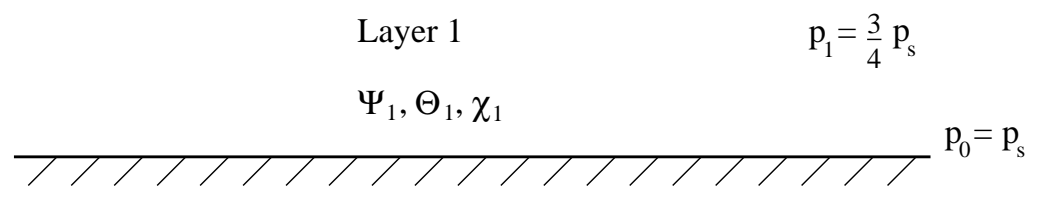

Eigure 1: The vertical discretisation in pressure coordinates. If the static stability, $\sigma$, is fixed, equations (4) and 5) determine $\Psi, \tau, \Theta$ and $\chi_{1}$.

The streamfunction and the potential temperature in the lower and the upper layer are denoted by $\Psi_{1,3}$ and $\Theta_{1,3}$, respectively. The pressure in the lower and the upper layer is set to $p_{1}=3 p_{s} / 4$ and $p_{3}=p_{s} / 4$. The equations are written in terms of vertical means and differences, defined as

$$
\begin{aligned}
\Psi=1 / 2\left(\Psi_{3}+\Psi_{1}\right) & \text { the barotropic streamfuction, } \\
\tau=1 / 2\left(\Psi_{3}-\Psi_{1}\right) & \text { the baroclinic streamfuction, } \\
\Theta=1 / 2\left(\Theta_{3}+\Theta_{1}\right) & \text { the mean potential temperature, } \\
\sigma=1 / 2\left(\Theta_{3}-\Theta_{1}\right) & \text { the static stability. }
\end{aligned}
$$

The static stability, $\sigma$, will be taken constant.

In addition to the conservative dynamics described in Lorenz [1960], we introduce linear damping through friction at the earth's surface, the terms proportional to $C$, friction at the boundary of the two layers, the term proportional to $C^{\prime}$ and Newtonian cooling, the term proportional to $h_{N}$. The temperature forcing is given by $\Theta^{*}$. The resulting equations are

$$
\begin{aligned}
\frac{\partial}{\partial t} \nabla^{2} \Psi & =-J\left(\Psi, \nabla^{2} \Psi+f\right)-J\left(\tau, \nabla^{2} \tau\right)-C \nabla^{2}(\Psi-\tau) \\
\frac{\partial}{\partial t} \nabla^{2} \tau & =-J\left(\tau, \nabla^{2} \Psi+f\right)-J\left(\Psi, \nabla^{2} \tau\right)+\nabla \cdot\left(f \nabla \chi_{1}\right)+C \nabla^{2}(\Psi-\tau)-2 C^{\prime} \nabla^{2} \tau \\
\frac{\partial}{\partial t} \Theta & =-J(\Psi, \Theta)+\sigma \nabla^{2} \chi_{1}-h_{N}\left(\Theta-\Theta^{*}\right)
\end{aligned}
$$

where the Jacobian operator, $J$, is defined as $J(A, B)=\nabla A \cdot \nabla B \times \mathbf{k}$ for any pair of functions $A, B$. The Coriolis parameter has been denoted by $f$.

Furthermore we have the thermal wind equation, relating the shear streamfunction to the mean potential temperature

$$
b c_{p} \nabla^{2} \Theta=\nabla \cdot(f \nabla \tau)
$$

Where $b=\frac{1}{2}\left[\left(\frac{p_{1}}{p_{s}}\right)^{\frac{c_{p}-c_{v}}{c_{p}}}-\left(\frac{p_{3}}{p_{s}}\right)^{\frac{c_{p}-c_{v}}{c_{p}}}\right] \approx 0.124$ comes out of the discretisation scheme in the vertical. 


\subsection{Domain and boundary conditions}

The set of equations (4) will be considered on an $f$-plane, a rectangular domain centered about a fixed latitude $\phi_{0}$ on which the Coriolis parameter is approximated by the constant value $f_{0}$. This domain has length $L$ in the zonal direction and $D$ in the meridional direction. On this plane we will use Cartesian coordinates $x \in[0, L)$ and $y \in[0, D]$. In the following we set $\phi_{0}=45^{\circ}$.

In the zonal direction we take periodic boundary conditions. In the meridional direction we have

$$
\frac{\partial \Psi}{\partial x}=\frac{\partial \tau}{\partial x}=\frac{\partial \chi_{1,3}}{\partial y}=0 \quad \text { at } y=0, D
$$

This means that there is no mass flux through the boundaries. The second condition was put forward by Philips [1954, and imposes that there is no net flow along the boundaries.

$$
\int_{0}^{L} \frac{\partial \Psi}{\partial y} \mathrm{~d} x=\int_{0}^{L} \frac{\partial \tau}{\partial y} \mathrm{~d} x=0 \quad \text { at } y=0, D
$$

It follows from the thermal wind equation (5), and the restriction that there be no net heat flux through the boundaries, that $\Theta$ satisfies

$$
\frac{\partial \Theta}{\partial x}=0 \quad \int_{0}^{L} \frac{\partial \Theta}{\partial y} \mathrm{~d} x=0 \quad \text { at } y=0, D
$$

With these boundary conditions we can consider $\Theta$ to describe deviations from the spatially averaged potential temperature.

\subsection{Scaling}

In table (11) the scales, suitable for the synoptic physics are listed. In the right column the numerical values, used below, are listed. In the following all quantities are dimensionless, unless otherwise indicated. The dimensionless length of the domain will be denoted by $s=L / D$.

\begin{tabular}{|l|l|l|}
\hline Length & $D$ & $5 \cdot 10^{3} \mathrm{~km}$ \\
\hline Time & $\Sigma^{-1}$ & 7 days \\
\hline Temperature & $R=\frac{f_{0} \Sigma D^{2}}{b c_{p}}$ & $34.3 \mathrm{~K}$ \\
\hline Mass & $M=\frac{p_{0} D^{2}}{2 g} \frac{f_{0}}{\Sigma} \frac{R}{\sigma}$ & $7.7 \cdot 10^{18} \mathrm{~kg}$ \\
\hline
\end{tabular}

Table 1: Scaling for the synoptic physics described by (4). In the right column the numerical values used.

\subsection{Equations for $\Psi$ and $\tau$}

Under the boundary conditions (6), the thermal wind relation (5) takes the simple form $\Theta=\tau$. This identity can be used to eliminate the velocity potential $\chi_{1}$ from equations (4.2) and (4.3). This results in a closed set of prognostic equations for $\Psi$ and $\tau$

$$
\begin{aligned}
& \frac{\partial}{\partial t} \nabla^{2} \Psi=-J\left(\Psi, \nabla^{2} \Psi\right)-J\left(\tau, \nabla^{2} \tau\right)-C \nabla^{2}(\Psi-\tau) \\
& \frac{\partial}{\partial t}\left(1-\alpha \nabla^{2}\right) \tau=-J(\Psi, \tau)+\alpha J\left(\tau, \nabla^{2} \Psi\right)+\alpha J\left(\Psi, \nabla^{2} \tau\right)-h_{N}\left(\tau-\tau^{*}\right) \\
&-\alpha C \nabla^{2}(\Psi-\tau)+2 \alpha C^{\prime} \nabla^{2} \tau
\end{aligned}
$$


where $\alpha=\sigma / f_{0}$ and $\tau^{*}=\Theta^{*}$. In this scaling $f_{0}^{-1} \approx 1.6 \cdot 10^{-2}$ is the Rossby number. We will study the lowest dimensional nontrivial spectral truncation of these equations.

\subsection{Energy}

In the absence of friction and forcing, the prognostic equations (7) conserve the sum of kinetic and available potential energy, defined respectively as

$$
K=\alpha \int_{0}^{1} \int_{0}^{s}(\nabla \Psi \cdot \nabla \Psi+\nabla \tau \cdot \nabla \tau) \mathrm{d} x \mathrm{~d} y \quad A=\int_{0}^{1} \int_{0}^{s} \Theta^{2} \mathrm{~d} x \mathrm{~d} y
$$

in units $M D^{2} \Sigma^{2}$. The simplified models will be shown to have a corresponding conserved quantity.

\section{The Galerkin approximation}

In order to approximate equations (7) by a finite number of ODE's we do a Galerkin projection onto Fourier modes. On this basis the variables are given by

$$
\begin{aligned}
\Psi(x, y, t) & =\sum_{n, m} \psi(m, n, t) \exp [\mathrm{i}(m k x+n l y)] \\
\tau(x, y, t) & =\sum_{n, m} \theta(m, n, t) \exp [\mathrm{i}(m k x+n l y)]
\end{aligned}
$$

where $k=2 \pi / s, l=\pi$. The boundary conditions, and the restriction that $\Psi$ and $\tau$ are real variables impose that

$$
\begin{aligned}
& \psi(m,-n)=-\psi(m, n) \quad \theta(m,-n)=-\theta(m, n) \quad \text { if } m \neq 0 \\
& \psi(0, n)=\psi(0,-n) \quad \theta(0, n)=\theta(0,-n) \\
& \psi(m, n)=\psi^{*}(-m,-n) \quad \theta(m, n)=\theta^{*}(-m,-n)
\end{aligned}
$$

This Fourier decomposition is equivalent to the introduction of a basis of eigenfunctions of the Laplacian operator on our domain, with the specified boundary conditions. The eigenfunctions are $\phi_{0 n}=\cos n l y$ for zonal wavenumber zero, and $\phi_{m n}=\mathrm{e}^{\mathrm{i} m k x} \sin n l y$ otherwise.

If we apply the zonally symmetric forcing $\theta^{*}=\frac{1}{2} \Delta T \phi_{01}$, with temperature contrast $\Delta T$ between the boundaries, there is an exact solution to equations (7). It is given by

$$
\Psi=\tau=\frac{1}{2} \frac{h_{N} \Delta T}{h_{N}+2 \alpha l^{2} C^{\prime}} \phi_{01}
$$

This solution is called the Hadley state and describes a strong jet in the upper layer, rising air at the south boundary and sinking air at the north boundary. The eigenfunctions with nonzero zonal wavenumber describe traveling waves which can grow if the Hadley circulation becomes dynamically unstable.

The projection of equations (7) is given by

$$
\begin{aligned}
\lambda_{c d} \dot{\psi}(c, d)= & -\lambda_{c d} C(\psi(c, d)-\theta(c, d)) \\
& +k l \sum_{p q r s} \lambda_{r s}(p s-q r) \delta(p+r-c) \mu(q+s-d)\{\psi(r, s) \psi(p, q)+\theta(r, s) \theta(p, q)\}
\end{aligned}
$$




$$
\begin{aligned}
& \bar{\lambda}_{c d} \dot{\theta}(c, d)=-h_{N}\left(\theta(c, d)-\theta^{*}(c, d)\right)-\alpha \lambda_{c d} C(\psi(c, d)-\theta(c, d))+2 \alpha C^{\prime} \lambda_{c d} \theta(c, d) \\
& +k l \sum_{p q r s}(p s-q r) \delta(p+r-c) \mu(q+s-d)\left\{1-\alpha\left(\left[p^{2}-r^{2}\right] k^{2}+\left[q^{2}-s^{2}\right] l^{2}\right)\right\} \psi(p, q) \theta(r, s)
\end{aligned}
$$

where $\delta$ is the Kronecker delta, $\theta^{*}$ is the Fourier transform of $\tau^{*}$ and $\mu$ is defined as

$$
\mu(a)=\int_{0}^{1} \mathrm{e}^{\mathrm{i} a l y} \mathrm{~d} y= \begin{cases}1 & \text { if } a=0 \\ 0 & \text { if } a \text { is even } \\ \frac{-2}{\pi \mathrm{i} a} & \text { if } a \text { is odd }\end{cases}
$$

The eigenvalues of the operators on the left hand side of equations (7) have been denoted by $\lambda_{a b}=-\left(a^{2} k^{2}+b^{2} l^{2}\right)$ and $\bar{\lambda}_{a b}=1-\alpha \lambda_{a b}$.

\section{The six dimensional truncation}

The smallest nontrivial truncation of equations (12) has six degrees of freedom. We set $s=2$ and define

$$
\begin{array}{lll}
x_{1}=2 \psi(0,1) & y_{1}=2 \theta(0,1) & T_{1}=2 \theta^{*}(0,1) \\
x_{2}=2 \sqrt{2} \operatorname{Re} \psi(1,1) & y_{2}=2 \sqrt{2} \operatorname{Re} \theta(1,1) & T_{2}=2 \sqrt{2} \operatorname{Re} \theta^{*}(1,1) \\
x_{3}=2 \sqrt{2} \operatorname{Im} \psi(1,1) & y_{3}=2 \sqrt{2} \operatorname{Im} \theta(1,1) & T_{3}=2 \sqrt{2} \operatorname{Im} \theta^{*}(1,1)
\end{array}
$$

These variables satisfy the following equations

$$
\begin{aligned}
\dot{x}_{1} & =-C\left(x_{1}-y_{1}\right) \\
\lambda_{11} \dot{x}_{2} & =-\lambda_{11} C\left(x_{2}-y_{2}\right)+\lambda_{10} \delta\left(x_{1} x_{3}+y_{1} y_{3}\right) \\
\lambda_{11} \dot{x}_{3} & =-\lambda_{11} C\left(x_{3}-y_{3}\right)-\lambda_{10} \delta\left(x_{1} x_{2}+y_{1} y_{2}\right) \\
\bar{\lambda}_{01} \dot{y}_{1} & =-\alpha \lambda_{01}\left(C x_{1}-\left[C+2 C^{\prime}\right] y_{1}\right)-h_{N}\left(y_{1}-T_{1}\right)+\delta\left(x_{3} y_{2}-x_{2} y_{3}\right) \\
\bar{\lambda}_{11} \dot{y}_{2} & =-\alpha \lambda_{11}\left(C x_{2}-\left[C+2 C^{\prime}\right] y_{2}\right)-h_{N}\left(y_{2}-T_{2}\right)+\delta\left(\bar{\lambda}_{10} x_{1} y_{3}-\nu_{10} x_{3} y_{1}\right) \\
\bar{\lambda}_{11} \dot{y}_{3} & =-\alpha \lambda_{11}\left(C x_{3}-\left[C+2 C^{\prime}\right] y_{3}\right)-h_{N}\left(y_{3}-T_{3}\right)-\delta\left(\bar{\lambda}_{10} x_{1} y_{2}-\nu_{10} x_{2} y_{1}\right)
\end{aligned}
$$

where $\delta=8 k l /(3 \pi)$ and $\nu_{a b}=1+\alpha \lambda_{a b}$. When we put dissipation and forcing to zero, the ODE system (15) has a conserved quantity $L$, defined by

$$
L=-\alpha \lambda_{01} x_{1}^{2}-\alpha \lambda_{11}\left(x_{2}^{2}+x_{3}^{2}\right)+\bar{\lambda}_{01} y_{1}^{2}+\bar{\lambda}_{11}\left(y_{2}^{2}+y_{3}^{2}\right)
$$

which corresponds to the projection of the sum of kinetic and available potential energy defined in equation (8). Using $L$ as a Lyapunov function we can show, that a trapping region for equations (15) is defined by

$$
L \leq \frac{3\|\mathbf{T}\|^{2}}{\left(h_{N}-2 \alpha \lambda_{11} C^{\prime}\right)^{2}}
$$

where $\|$.$\| denotes the L_{2}$ norm, and we have assumed that $0<h_{N} \leq C, 0<2 C^{\prime} \leq C$ and $\sigma \leq 1$, a realistic range for the parameters. Note, that the divergence of the vector field, defined by equation (15), is constant and negative. Therefore, volume elements always shrink. 


\subsection{Bifurcation diagram}

In figure (2) the partial bifurcation diagram of system (15) is shown. This diagram, and all other diagrams below, have been calculated using the software package AUTO (Doedel et al. [1986]). To obtain this picture, we varied $T_{1}$ and $T_{2}$, setting $T_{3}=0$. Due to a discrete symmetry in equations (15), given by

$$
\begin{aligned}
& \mathbf{x} \rightarrow \mathbf{x}^{\prime}=\mathbf{R x} \\
& \mathbf{y} \rightarrow \mathbf{y}^{\prime}=\mathbf{R y} \\
& \mathbf{T} \rightarrow \mathbf{T}^{\prime}=\mathbf{R T}
\end{aligned} \quad \text { where } \mathbf{R}=\left(\begin{array}{lll}
1 & 0 & 0 \\
0 & 0 & -1 \\
0 & 1 & 0
\end{array}\right)
$$

setting $T_{2}=0$ and varying $T_{3}$ yields the same diagram. This symmetry corresponds to a translation $x \rightarrow x^{\prime}=x+1 / 2$ in the physical domain. Furthermore, we have set $C=3.5, h_{N}=0.7, C^{\prime}=0.5$ and $\sigma=0.9$. This corresponds to a damping time scale of two days at the earth's surface and two weeks at the layer interface. The thermal damping time scale is ten days and the temperature difference between the layers is about $34 \mathrm{~K}$.

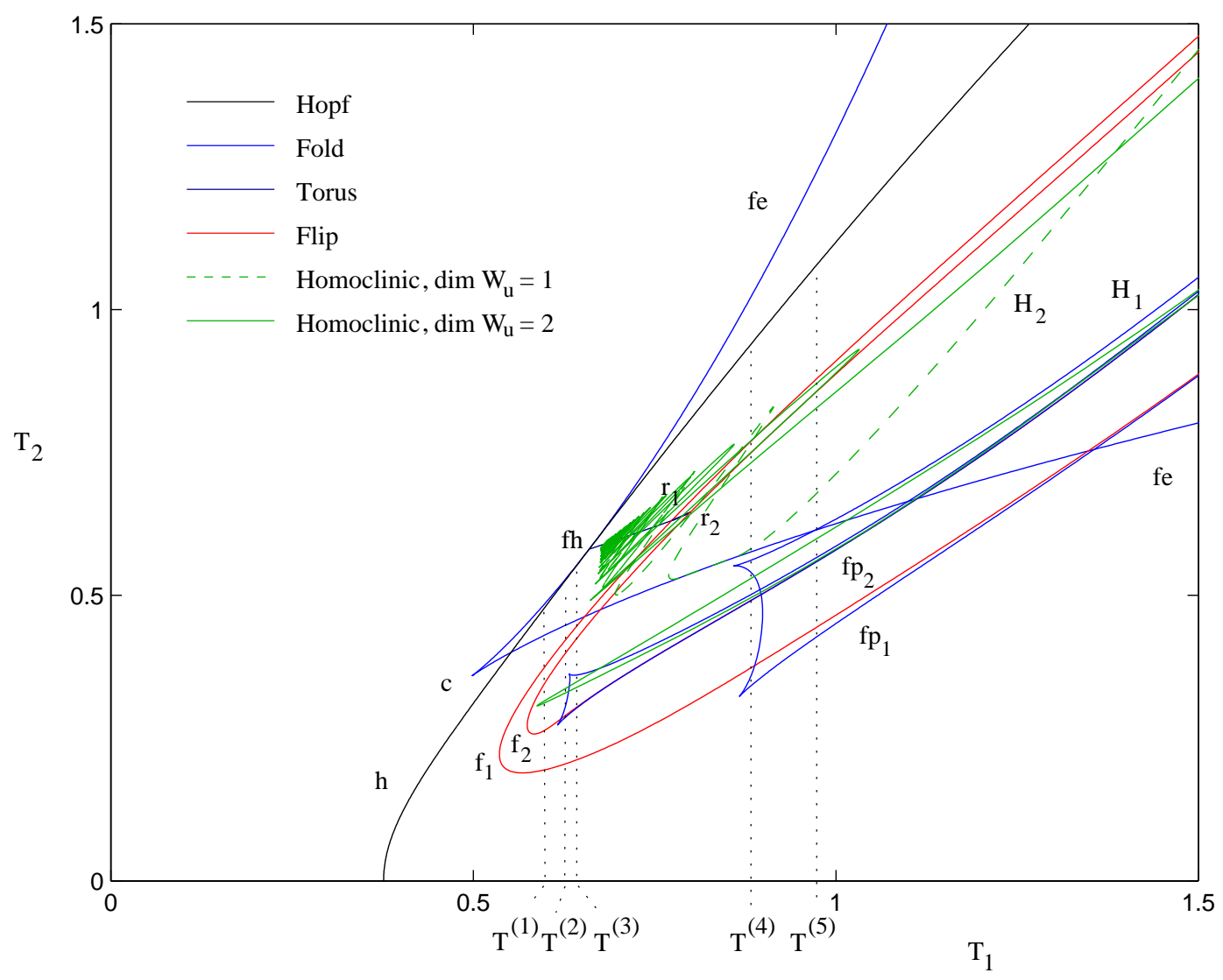

Figure 2: Bifurcation diagram of system (15). The dotted lines at $T_{1}=T^{(1 \ldots 5)}$ refer to figures (4)-(6).

If we put $T_{2}=0$ and increase $T_{1}$ from zero, at first a stable equilibrium is the unique limit set in the phase space of system (15). This equilibrium corresponds to the Hadley state (11). At the Hopf bifurcation line, marked $\mathrm{h}$, this equilibrium becomes unstable and a stable periodic orbit is 
created. This is our model's baroclinic instability. The periodic orbit corresponds to a traveling baroclinic wave.

The two line segments fe, joint at cusp point c, denote a fold bifurcation of the equilibrium. Within the V-shaped region, bounded by curve fe, there are three equilibria, one of which is stable. At the codimension two point, marked fh, the Hopf line and the fold line are tangent. At this point an equilibrium exists with one zero eigenvalue and a complex pair on the imaginary axis. The unfolding of this point can be found in Kuznetsov [1998. In the following, we will adopt the notation of this book for normal form coefficients. An algorithm for computing the normal form coefficients of fold-Hopf points is described in Kuznetsov [1999 and implemented in the forthcoming release of CONTENT (Kuznetsov and Levitin [1997]). In this case, we have normal form coefficients $s=1$ and $\theta<0$.

A torus bifurcation line emerges from point fh, and connects to the flip bifurcation line marked $\mathrm{f}_{1}$. Above the torus bifurcation line, the periodic orbit created on Hopf curve $\mathrm{h}$ is unstable, below the torus bifurcation line it is stable and coexists with a saddle type, two dimensional torus. At the point where the torus and the flip bifurcation lines meet, the periodic orbit has two Floquet multipliers equal to minus unity. This point is marked $r_{1}$ for $1: 2$ resonance. At this point, we have normal form coefficient $s=-1$. Connected to the resonance point $\mathrm{r}_{1}$ there is torus bifurcation line of the period doubled orbit, which leads to another $1: 2$ resonance point, $\mathrm{r}_{2}$. In fact, flip bifurcation lines $f_{1}$ and $f_{2}$ are the first two of a period doubling cascade. There seems to be a accumulation of $1: 2$ resonance points directly to the right of $\mathrm{r}_{2}$. Such an accumulation has been described in the context of a biological model in Kuznetsov [1998], chapter 9.6. Wieczorek et al. [2001] found it in a rate equation model for a semiconductor laser. They also provide a partial unfolding of this codimension two phenomenon.

From codimension two point fh two homoclinic bifurcation lines emanate. Both homoclinic connections are attached to a saddle focus. Along $\mathrm{H}_{2}$ the unstable manifold of the saddle focus is one dimensional and the saddle value, $\sigma$, is negative. On this line a stable periodic orbit is created. Along $\mathrm{H}_{1}$, the saddle focus has a complex pair of eigenvalues with positive real part. The saddle value is positive along the larger part $\mathrm{H}_{1}$ and another stable periodic orbit is created. However, near the leftmost turning point of this curve, the saddle value changes sign. On a small segment it is negative, indicating that Shil'nikov type chaos can occur. In the literature, this type of Shil'nikov bifurcation, with complex unstable leading eigenvalues, is uncommon.

The points, where the saddle value is zero, are called neutral saddle focus, or Belyakov, transitions. What is known about the unfolding of this transition is summed up in Champneys and Kuznetsov [1994]. In figure (3) the transition points are shown in more detail, along with a phase portrait. An infinite number of cusps of fold lines of periodic orbits are expected to accumulate here, corresponding to the creation of successive folds of the branch of periodic solutions which becomes homoclinic on $\mathrm{H}_{1}$. However, as described in Glendinning and Sparrow [1984, the neutral saddle focus transition is continuous, and these cusp bifurcations correspond to orbits of very high period which are hard to detect numerically. Looking at sections like in figure (5), the case with negative saddle values cannot be discerned from the case with positive saddle value.

In figure (4) a cross section of diagram (2) is shown. We have fixed $T_{1}=T^{(1)}$ and let $T_{2}$ vary as indicated in diagram (2). The top picture shows a number of branches of the period doubling cascade, as well as the primary homoclinic branch corresponding to curve $\mathrm{H}_{1}$. The bottom picture, on the same horizontal scale, is a limit point diagram. This picture was obtained by calculating the Poincaré map on the plane $\mathcal{S}=\left\{(\mathbf{x}, \mathbf{y}) \in \mathbb{R}^{6} \mid y_{2}=0\right\}$. After a sufficiently long integration, to filter out transients, the value of $y_{1}$ was plotted at a number of iterations of the Poincaré map. The behaviour is chaotic between the accumulation points of the period doubling cascade and changes qualitatively near the Shil'nikov bifurcation.

The lines marked $\mathrm{fp}_{1}$ and $\mathrm{fp}_{2}$ denote fold bifurcations of periodic orbits. Near the cusps of 

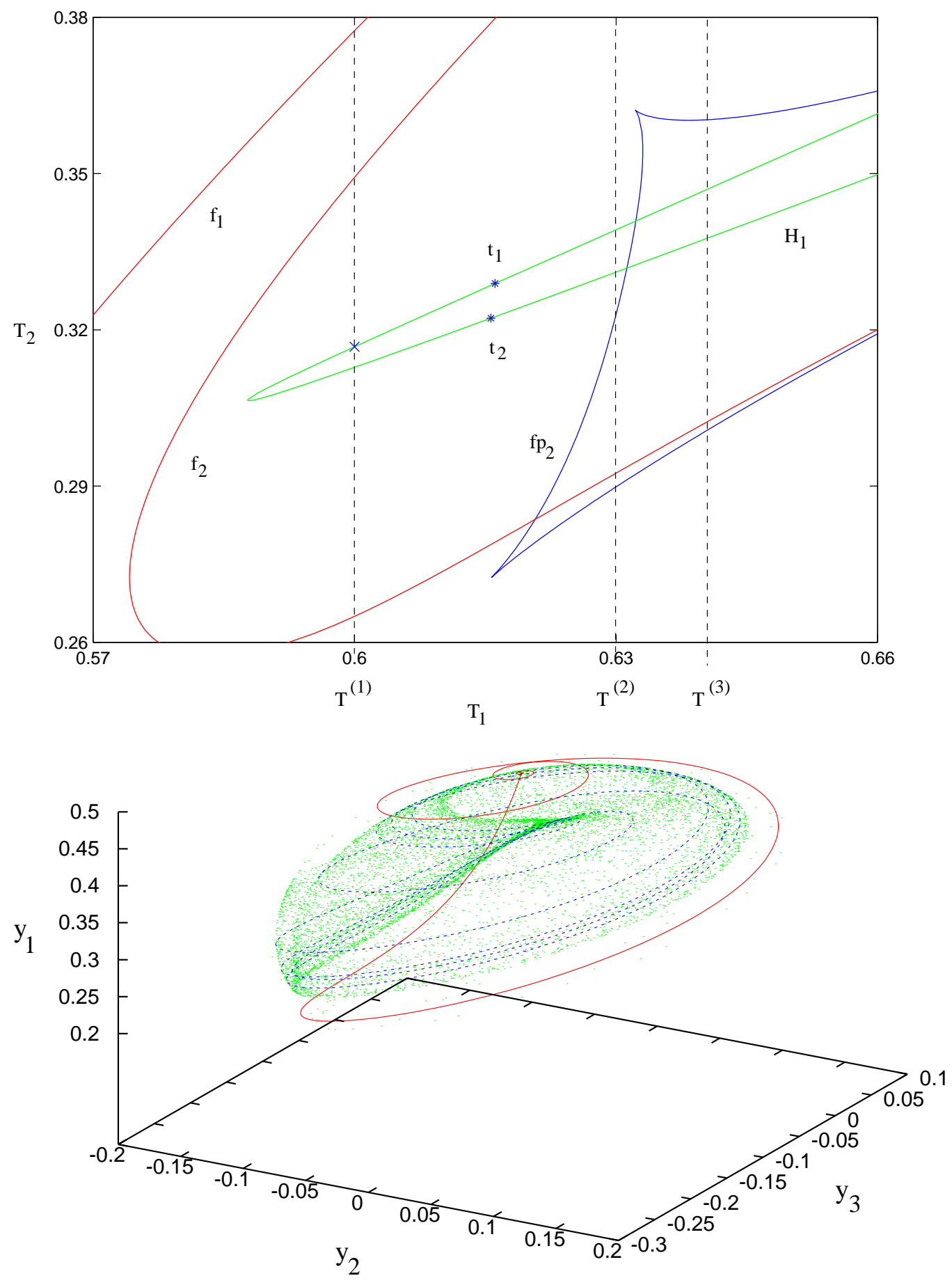

Figure 3: Top: detail of bifurcation diagram (2). The neutral saddle focus transitions have been marked $t_{1,2}$. To the left of these transitions the saddle value is negative and the Shil'nikov condition is satisfied. Bottom: phase portrait at $\left(T_{1}, T_{2}\right)=(0.6,0.3168)$. These parameter values have been marked with a cross in the top picture. In red: the homoclinic orbit. In blue, dashed: the periodic solution after four period doublings, i.e. the fifth branch in the top picture of figure (4). In green: points on the chaotic attractor. 

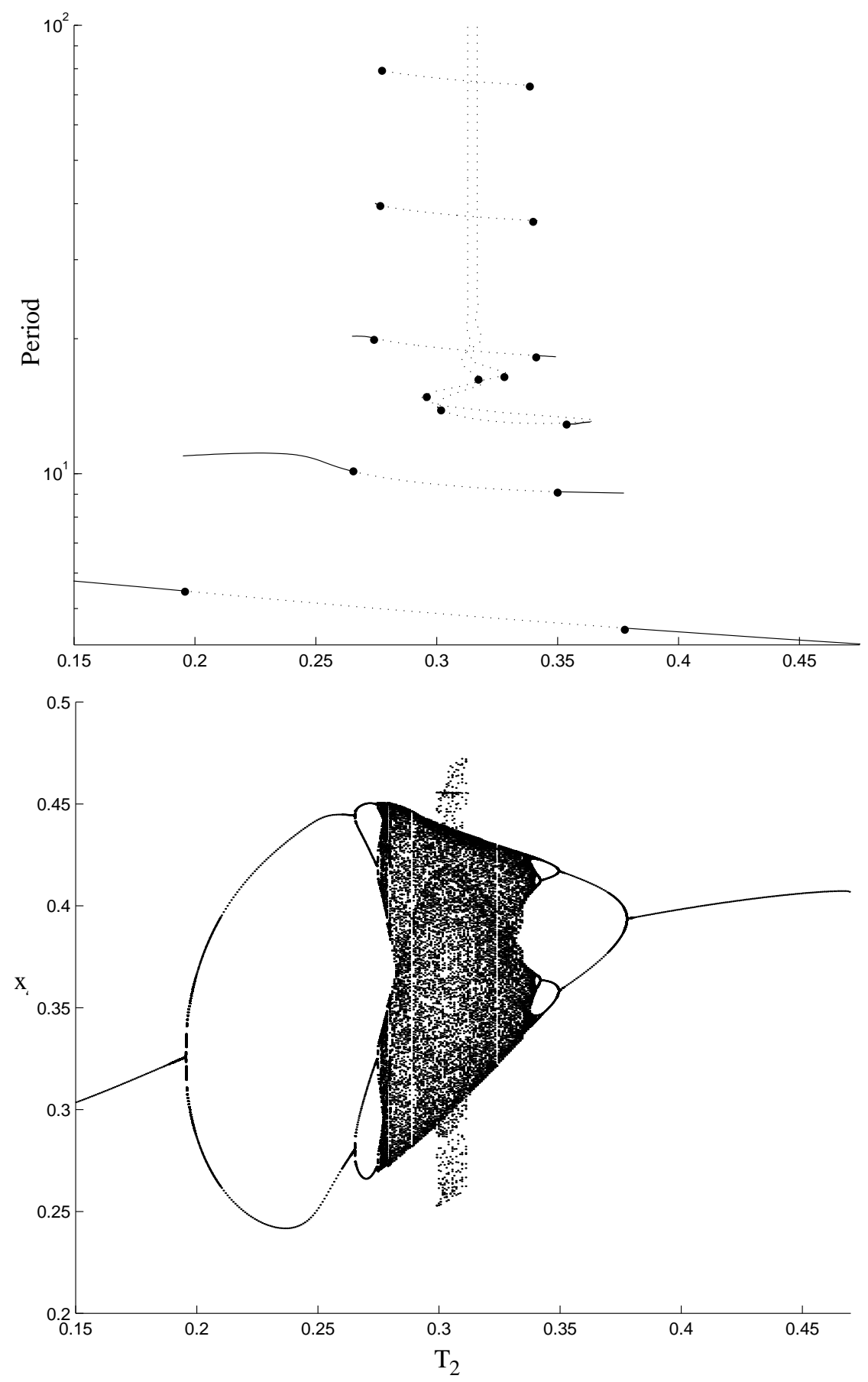

Figure 4: Top: continuation of the periodic orbit created on curve $\mathrm{h}$, along the line $T_{1}=T^{(1)}$ in figure (2). Solid lines denote stable branches, dotted lines denote unstable branches, Hopf bifurcation point are marked with dots. Also shown is the primary homoclinic branch corresponding to curve $\mathrm{H}_{1}$. Bottom: limit point diagram of the Poincaré map on $\mathcal{S}$. 

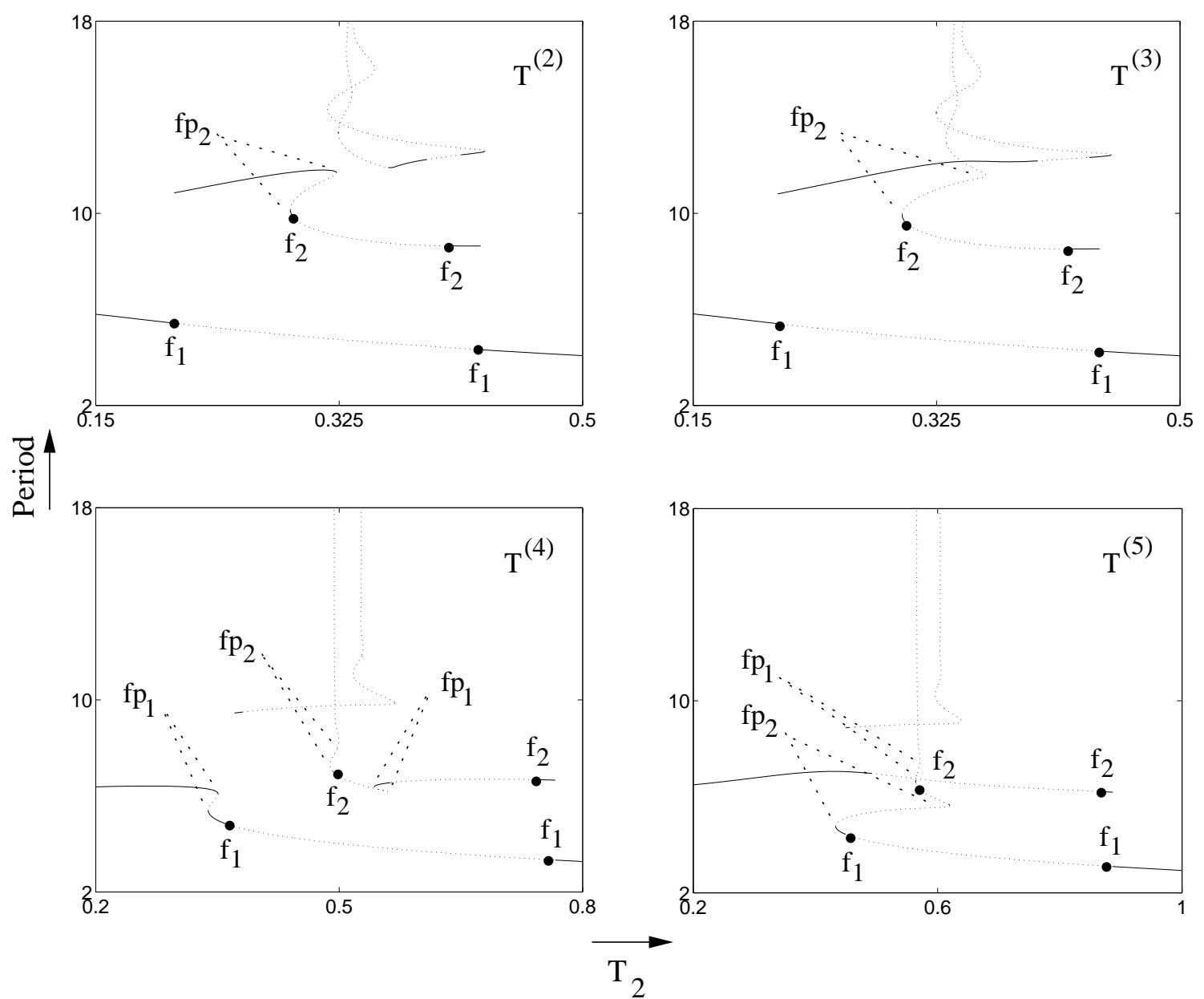

Figure 5: Cross sections of diagram (2). Dots denote flip bifurcations, solid lines stable branches and dotted lines unstable branches. The labels $\mathrm{f}_{1,2}$ and $\mathrm{fp}_{1,2}$ refer to diagram (2). Top left: $T_{1}=T^{(2)}=0.63$. The basic cycle, born on Hopf line $\mathrm{h}$, and its period double version, are not connected to the homoclinic branch. Top right: $T_{1}=T^{(3)}=0.64$. The period doubled cycle now becomes homoclinic on $\mathrm{H}_{1}$. Bottom left: $T_{1}=T^{(4)}=0.88$. The branch of the basic cycle folds. Bottom right: $T_{1}=T^{(5)}=0.98$. From one end, $T_{2}$ increasing from zero, the branch of the basic cycle ends in a period halving bifurcation. From the other end, $T_{2}$ decreasing, the branch of the basic cycle becomes homoclinic on $\mathrm{H}_{1}$.

these fold lines, the periodic orbit, created at Hopf line h, and its period doubled versions, switch branches with periodic orbits that become homoclinic near $\mathrm{H}_{1}$. This process is illustrated in figure (5). In the top left picture, where we have $T_{1}=T^{(2)}$, none of the branches of the period doubling cascade are connected to the homoclinic branch, see also figure (画(top). For $T_{1}>T^{(2)}$, however, the first period doubled branch becomes homoclinic on curve $\mathrm{H}_{1}$. The period doubled branch and the homoclinic branch collide in a transcritical bifurcation. This process is repeated for the branch of the basic cycle, continued from Hopf line $h$. Therefore, the flip bifurcation lines $f_{1,2, \ldots}$ do not simply form a cascade and an inverse cascade. At the fold lines $\mathrm{fp}_{1,2}$ the simple structure of figure (函)(top) at $T_{1}=T^{(1)}$ is rearranged.

Summarising, the qualitative dynamics of system (15) is as follows. Left of the Hopf line h, and within the V-shaped region bounded by fold curve fe, there is a stable equilibrium. To the 
right of curve $\mathrm{h}$, and below curve fe, the behaviour is periodic before crossing flip bifurcation curve $f_{1}$. When crossing $f_{1}$ near the leftmost fold of the homoclinic curve $H_{1}$, where the saddle value is positive, a combination of period doubling and Shil'nikov chaos is encountered, as demonstrated in figures (3) and (4). To the right of the neutral saddle focus transition points, the behaviour is alternatingly periodic and chaotic. Due to the branch switching, shown in figure (5), the parameter space is divided into small chaotic and periodic windows.

In figure (6) the Kaplan-Yorke dimension of the attractor is shown for $T_{1}=T^{(1)}$ and values of $T_{2}$ at which complex dynamics arise. Most remarkably, the attractor dimension does not exceed three. Also, the equilibria and periodic orbits studied in figure (2) have a feature in common. They all have three strongly contracting directions. These observations suggest, that the dynamics of system (15) take place on a three dimensional invariant manifold. In the next section we will present an approximate, three dimensional invariant manifold of this system, which enables us to reduce the model to three degrees of freedom.

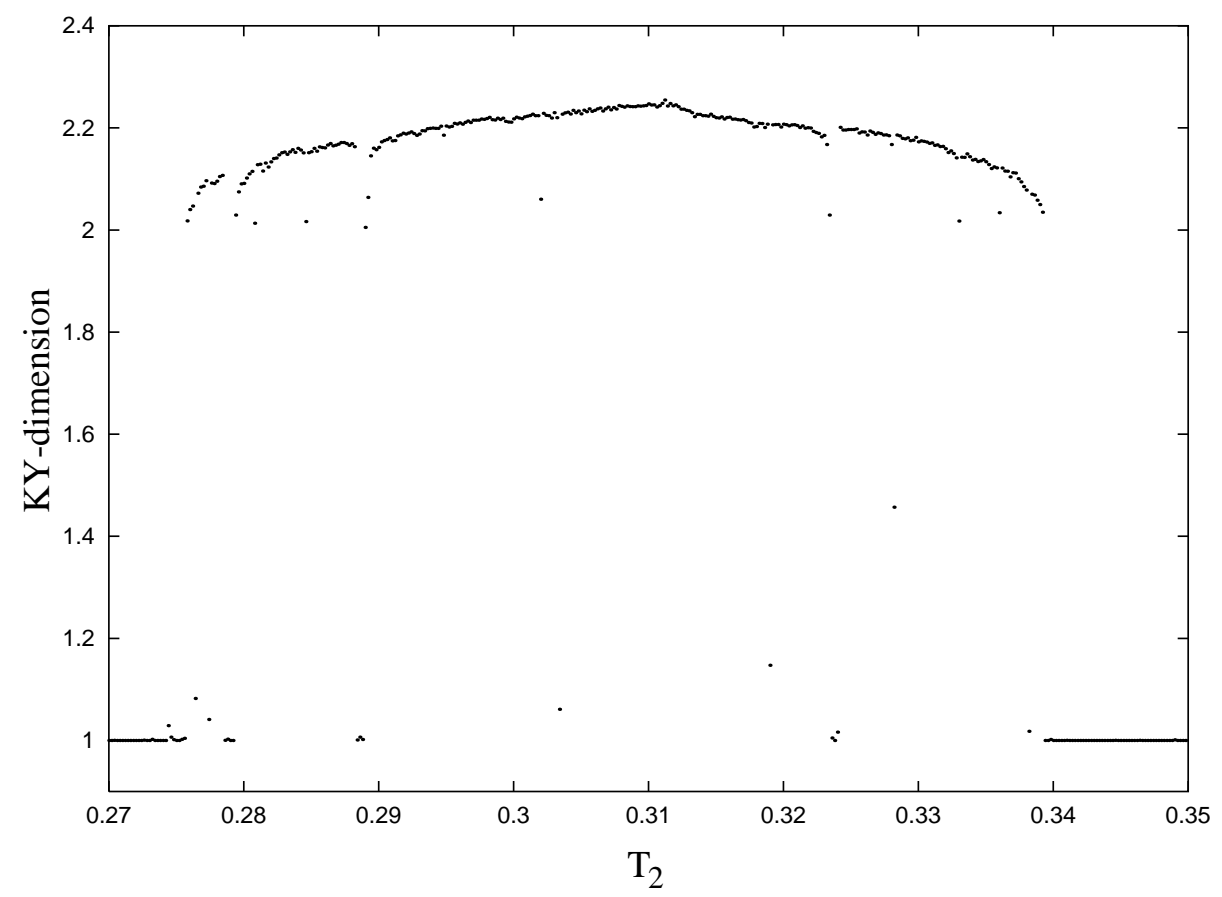

Figure 6: Numerical estimate of the Kaplan-Yorke dimension, obtained from an integration during $\Delta t=1.5 \cdot 10^{4}$ at each parameter value $T_{2}$, for fixed $T_{1}=T^{(1)}$.

\subsection{Reduction to an invariant manifold}

With the parameters set to the values, specified in section (4.1), we can scale the constants in equations (15.1)-(15.3) as

$$
\bar{C}=\epsilon C \quad \kappa=\epsilon \frac{\delta \lambda_{10}}{\lambda_{11}}=\epsilon \frac{\delta}{2}
$$


where $\epsilon \approx 1 / 4$ and $\bar{C} \approx \kappa \approx 1$. System (15) is then written symbolically as

$$
\begin{aligned}
\epsilon \dot{\mathbf{x}} & =\overline{\mathbf{f}}(\mathbf{x}, \mathbf{y}) \\
\dot{\mathbf{y}} & =\mathbf{g}(\mathbf{x}, \mathbf{y})
\end{aligned}
$$

where $\overline{\mathbf{f}}$ is defined as

$$
\begin{aligned}
& \bar{f}_{1}=-\bar{C}\left(x_{1}-y_{1}\right) \\
& \bar{f}_{2}=-\bar{C}\left(x_{2}-y_{2}\right)+\kappa\left(x_{1} x_{3}+y_{1} y_{3}\right) \\
& \bar{f}_{3}=-\bar{C}\left(x_{3}-y_{3}\right)-\kappa\left(x_{1} x_{2}+y_{1} y_{2}\right)
\end{aligned}
$$

We assume, that there exists a globally attracting, three dimensional invariant manifold in system (20), denoted by $W_{\epsilon}$. This manifolds is represented as the graph of a function, $\phi_{\epsilon}$, of the baroclinic components:

$$
W_{\epsilon}=\left\{(\mathbf{x}, \mathbf{y}) \in \mathbb{R}^{6} \mid \mathbf{x}=\phi_{\epsilon}(\mathbf{y})\right\}
$$

and satisfies

$$
\overline{\mathbf{f}}\left(\phi_{\epsilon}, \mathbf{y}\right)=\epsilon \mathrm{D} \phi_{\epsilon} \cdot \mathbf{g}\left(\phi_{\epsilon}, \mathbf{y}\right)
$$

The solution of (23) can be approximated asymptotically. Substituting the regular expansion $\phi_{\epsilon}=\phi_{0}+\epsilon \phi_{1}+\ldots$, we find

$$
\phi_{0}(\mathbf{y})=\left(\begin{array}{ccc}
1 & 0 & 0 \\
0 & A\left(y_{1}\right) & B\left(y_{1}\right) \\
0 & -B\left(y_{1}\right) & A\left(y_{1}\right)
\end{array}\right) \mathbf{y}
$$

where $A$ and $B$ are defined as

$$
A\left(y_{1}\right)=\frac{\bar{C}^{2}-\kappa^{2} y_{1}^{2}}{\bar{C}^{2}+\kappa^{2} y_{1}^{2}}=\cos \gamma \quad \text { and } \quad B\left(y_{1}\right)=\frac{2 \bar{C} \kappa y_{1}}{\bar{C}^{2}+\kappa^{2} y_{1}^{2}}=\sin \gamma
$$

This zeroeth order approximation has a clear physical interpretation. It describes a zonally symmetric part of the flow which is equivalent barotropic (i.e. $\Psi_{3} \propto \Psi_{1}$ ) and a phase shift, $\gamma$, between the traveling waves in the upper and the lower layer.

The nonlinear terms in equations (15) can be divided into two groups: one represents advection of waves with the zonally symmetric flow (the terms proportional to $x_{1}$ ) and the other represents energy exchange between waves and the zonally symmetric flow. The nonlinear terms in equation (15.4) belong to the second group. If the phase shift is zero, these terms cancel, and the wave components are decoupled from the zonally symmetric components. Thus, the function $\phi_{0}$ describes how the energy transfer depends on strength of the zonally symmetric flow.

The asymptotic expansion of $\phi_{\epsilon}$ can not accurately describe the solution of equation (23) at the realistic parameter value $\epsilon=0.25$. Therefore, we use a numerical algorithm to continue the solution, known analytically in the limit of $\epsilon \downarrow 0$. The algorithm is similar to the graph transform described in Broer et al. [1997]. In contrast to the systems considered in their work, however, ours is a continuous time system. Therefore, we apply the graph transform to the map, induced by an implicit Euler step which approximates the flow of system (20) over a finite time interval. This approach was already used by Foias et al. [1988] to approximate inertial manifolds by their modified Galerkin method.

Another difference is the choice of coordinates. Here, we do not represent the (approximate) invariant manifold as the graph of a section of the normal bundle of some given approximation. 


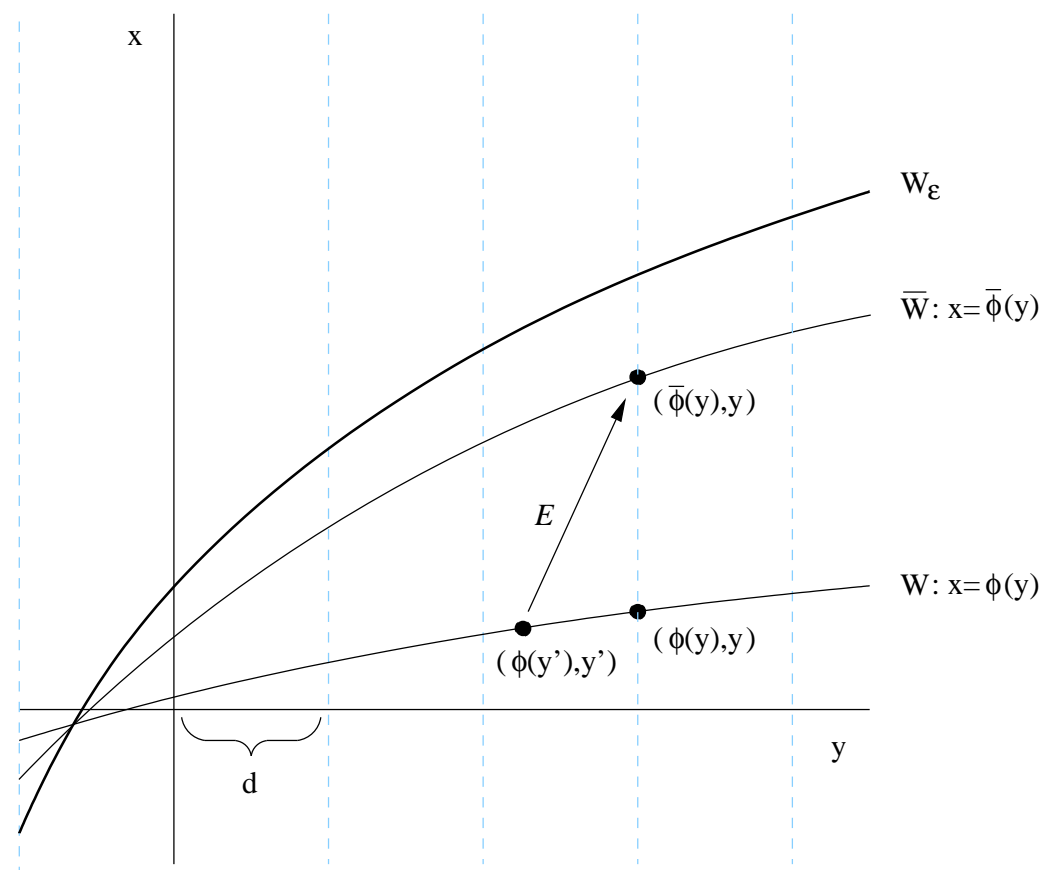

Figure 7: Schematic picture of the graph transform. Note, that equilibria of system (20) are intersection points of $W, \bar{W}$ and $W_{\epsilon}$. The dashed lines represent the mesh $\mathbf{y}_{i j k}$.

Instead, we globally represent it as the graph of a function $\phi_{\epsilon}(\mathbf{y})$. This can be done provided that $\mathrm{D} \phi_{\epsilon}$ has full rank. For small $\epsilon$ this condition is satisfied, as we have $\operatorname{det} \mathrm{D} \phi_{\epsilon}=1+\mathrm{O}(\epsilon)$. When increasing $\epsilon$ the condition has to be checked numerically.

Suppose that, for some fixed value of $\epsilon$, we have an approximation, $W$, of $W_{\epsilon}$. By assumption, $W_{\epsilon}$ is globally attracting so that the image of $W$ under the flow over a finite time interval of system (20) lies closer to $W_{\epsilon}$ than $W$. Thus, by approximating the flow of system (20), we can calculate an improved approximation $\bar{W}$. For this end we use the map $E: \mathbb{R}^{6} \rightarrow \mathbb{R}^{6}$, where $(\overline{\mathbf{x}}, \overline{\mathbf{y}})=E((\mathbf{x}, \mathbf{y}))$ is the solution of

$$
\begin{aligned}
& \overline{\mathbf{x}}=\mathbf{x}+\frac{\Delta}{\epsilon} \overline{\mathbf{f}}(\overline{\mathbf{x}}, \overline{\mathbf{y}}) \\
& \overline{\mathbf{y}}=\mathbf{y}+\Delta \mathbf{g}(\overline{\mathbf{x}}, \overline{\mathbf{y}})
\end{aligned}
$$

which defines an implicit Euler time step. The step size, $\Delta$, is a free parameter. The improved approximation is then defined as $\bar{W}=E(W)$.

In order to represent $\bar{W}$ as the graph of a function $\bar{\phi}(\mathbf{y})$, we map a point $(\phi(\mathbf{y}), \mathbf{y}) \in W$ onto the point $(\bar{\phi}(\mathbf{y}), \mathbf{y}) \in \bar{W}$, where $\bar{\phi}$ is the solution of

$$
\bar{\phi}=\phi(\mathbf{y}-\Delta \mathbf{g}(\bar{\phi}, \mathbf{y}))+\frac{\Delta}{\epsilon} \overline{\mathbf{f}}(\bar{\phi}, \mathbf{y})
$$

In other words, for a given vector $\mathbf{y}$ we look for the point $\left(\phi\left(\mathbf{y}^{\prime}\right), \mathbf{y}^{\prime}\right) \in W$ which is mapped according to $(26)$ onto $(\bar{\phi}(\mathbf{y}), \mathbf{y}) \in \bar{W}$. In figure (7) this procedure is sketched.

Differentiating equation (27) with respect to $\mathbf{y}$, we find

$$
\left(\mathbb{I}-\frac{\Delta}{\epsilon} \mathrm{D}_{x} \overline{\mathbf{f}}+\left.\Delta \mathrm{D} \phi\right|_{\mathbf{y}^{\prime}} \mathrm{D}_{x} \mathbf{g}\right) \mathrm{D}_{y} \bar{\phi}=\left.\mathrm{D} \phi\right|_{\mathbf{y}^{\prime}}\left(\mathbb{I}-\Delta \mathrm{D}_{y} \mathbf{g}\right)+\frac{\Delta}{\epsilon} \mathrm{D}_{y} \overline{\mathbf{f}}
$$


from which we can calculate $\mathrm{D}_{y} \bar{\phi}$, and thus the tangent space $T_{(\bar{\phi}, \mathbf{y})} \bar{W}$, from $\mathrm{D}_{y} \phi$ and the Jacobian of system (20). This enables us to define a local error function. Let $F=(\overline{\mathbf{f}} / \epsilon, \mathbf{g})$ denote the vector field $(20)$ and $\mathcal{P}$ the orthogonal projection onto the tangent space at $(\bar{\phi}, \mathbf{y})$. Then we define the error function

$$
e(\mathbf{y})=\frac{2}{\pi} \arccos \frac{\|\mathcal{P} F(\bar{\phi}, \mathbf{y})\|}{\|F(\bar{\phi}, \mathbf{y})\|}
$$

We will represent the approximate invariant manifold on a cubic lattice in $\mathbb{R}^{3}$. The vertices of this lattice are located at $\mathbf{y}_{i j k}=d(i, j, k)^{T}$, where $\mathrm{d}$ is the lattice spacing and $i, j, k$ are integers. We consider a finite number of lattice points, demanding that all points $\left(\phi_{0}\left(\mathbf{y}_{i j k}\right), \mathbf{y}_{i j k}\right)$ lie within the trapping region defined in section (41). In fact, inequality (17) is a fairly course estimate. In order to reduce computation time and data storage, we use a sharper estimate, obtained by numerical computation of the eigenvalues of the linear part of equations (15.1-15.6). To an approximate solution $\bar{\phi}$ we can then assign the error

$$
\mathcal{E}=\max _{i j k} e\left(\mathbf{y}_{i j k}\right)
$$

In order to approximate the invariant manifold for finite $\epsilon$, we will proceed in steps. First of all, we fix an initial value for $\epsilon$ and calculate $\phi_{1}$ and $\mathrm{D} \phi_{1}$ on each lattice point. Next, we solve equation (27), by Newton iteration, and subsequently (28), to find the next approximation $\bar{\phi}$ and its derivatives. A suitable initial guess for the Newton iteration is obtained by linearisation in $\xi=\mathbf{y}^{\prime}-\mathbf{y}$. This yields

$$
\left(\begin{array}{c}
\bar{\phi}(\mathbf{y}) \\
\mathbf{y}
\end{array}\right)=E\left(\begin{array}{c}
\phi\left(\mathbf{y}^{\prime}\right) \\
\mathbf{y}^{\prime}
\end{array}\right) \approx E\left(\begin{array}{c}
\phi(\mathbf{y}) \\
\mathbf{y}
\end{array}\right)+\mathrm{D} E \cdot\left(\begin{array}{c}
\mathrm{D} \phi \\
\mathbb{I}
\end{array}\right) \cdot \xi
$$

from which we can estimate $\xi$ and, subsequently, $\bar{\phi}$. The graph transform is iterated untill the global error is smaller than a fixed threshold $\mathcal{E}_{\max }$. If this is achieved, we increase epsilon and iterate the process.

When solving equation (27), evaluation of $\phi$ and $\mathrm{D} \phi$ inbetween lattice points is necessary. This is done by linear interpolation. To this end, each cube in the lattice is divided into six tetraeders of equal volume. The associated error is expected to be of order $\mathrm{O}\left(d^{2}\right)$. At the edge of the domain we consider, it may happen, that evaluation of $\phi$ outside this domain is required. If so, we solve equation (27), substituting $\phi\left(\mathbf{y}^{\prime}\right) \rightarrow \phi(\mathbf{y})+\mathrm{D} \phi \cdot \xi$. The derivatives of $\bar{\phi}$ are then calculated by finite difference. This, however, introduces an error, which disables us to continue the invariant manifold up to $\epsilon=0.25$.

For example we approximate the manifold at parameter values $\left(T_{1}, T_{2}\right)=(0.6,0.25)$. The step size is fixed to $\Delta=0.0025$ and the increment of $\epsilon$ is chosen in the range [0.001,0.005]. The maximal error is fixed to $\mathcal{E}_{\max }=0.05$. At each value of $\epsilon$ about six iterations of the graph transform are needed. The computations were done with a lattice spacing of $d=0.01$, in the trapping region $L \leq 0.52$. Thus, about $5 \cdot 10^{5}$ points on the manifold are calculated.

In figure (8) the approximate invariant manifold is illustrated. Shown are the stable periodic orbit of system (20) at $\epsilon=0.1$ and $\left(T_{1}, T_{2}\right)=(0.6,0.25)$ and a forward integration of the system $\dot{\mathbf{y}}=\mathbf{g}\left(\phi_{\epsilon}(\mathbf{y}), \mathbf{y}\right)$. To find the latter integral curve we approximated every point of $\phi_{\epsilon}(\mathbf{y})$ needed by linear interpolation of the results of the calculation presented above.

\section{The reduced system}

In the following, we will describe system (20), reduced to the approximate invariant manifold $W_{0}$. This reduction can be done analytically, so that we can compare bifurcation diagrams. Physically, 

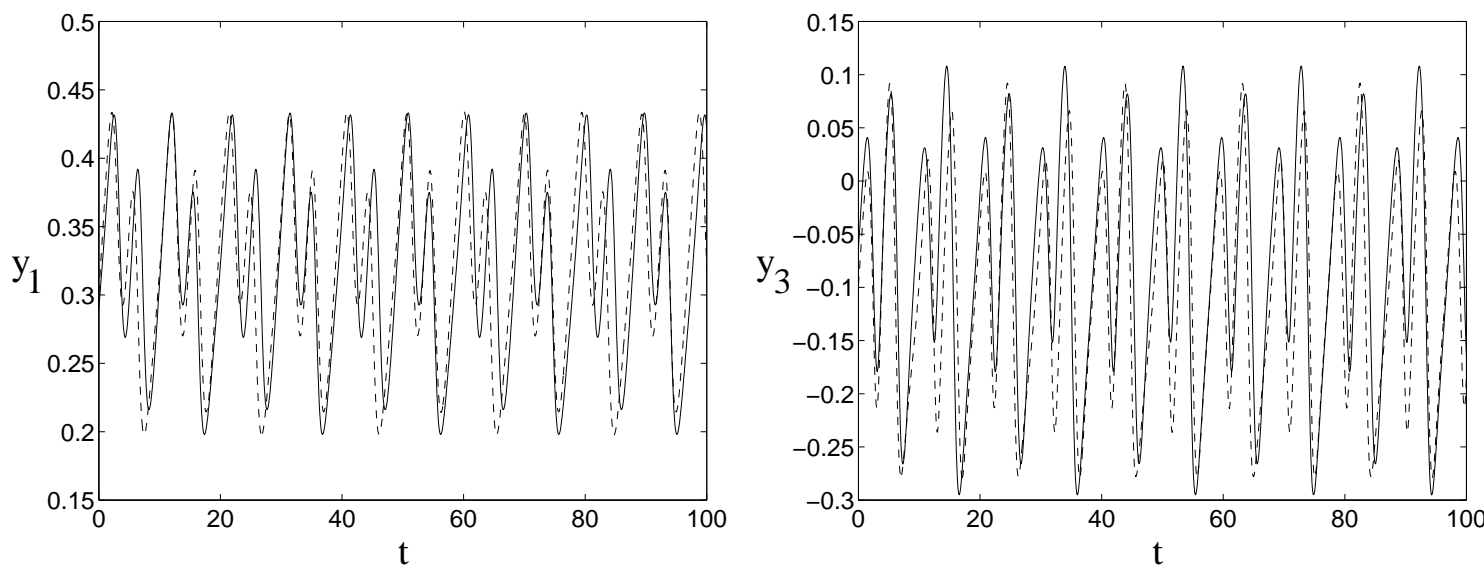

Figure 8: Time series of the zonally symmetric component, $y_{1}$, and a wave component, $y_{3}$. Solid: solution of system (20) at $\left(T_{-} T_{2}\right)=(0.6,0.25)$ and $\epsilon=0.1$. Dashed: solution of $\dot{\mathbf{y}}=\mathbf{g}\left(\phi_{\epsilon}(\mathbf{y}), \mathbf{y}\right)$, using the approximate solution $\phi_{\epsilon}$ of equation (23) obtained by the graph transform method.

an argument to study the system reduced to $W_{0}$, rather than to the numerically approximated $W_{\epsilon}$, is that the quantitative error, introduced by setting $\epsilon=0$, is smaller than the error introduced by the Galerkin approximation. Equations (15) form a qualitative model of one aspect of the atmospheric circulation, namely the interaction between the jet stream and the baroclinic waves. A further simplification is justified if it keeps the qualitative behaviour in tact.

Substituting expression (24), we find that the reduced system, $\dot{\mathbf{y}}=\mathbf{g}\left(\phi_{0}(\mathbf{y}), \mathbf{y}\right)$, is given by

$$
\begin{aligned}
& \dot{y}_{1}=-c_{1} y_{1}-d_{1}\left(y_{2}^{2}+y_{3}^{2}\right)+\bar{T}_{1} \\
& \dot{y}_{2}=-c_{2} y_{2}+c_{3} y_{3}+d_{3} y_{1} y_{3}+d_{2} y_{1} y_{2}+\bar{T}_{2} \\
& \dot{y}_{3}=-c_{2} y_{3}-c_{3} y_{2}-d_{3} y_{1} y_{2}+d_{2} y_{1} y_{3}+\bar{T}_{3}
\end{aligned}
$$

where we have introduced

$$
\begin{array}{lll}
c_{1}=\left(-2 \alpha \lambda_{01} C^{\prime}+h_{N}\right) / \bar{\lambda}_{01} & \bar{T}_{1}=h_{N} T_{1} / \bar{\lambda}_{01} & d_{1}=\delta B / \bar{\lambda}_{01} \\
c_{2}=\left(-\alpha \lambda_{11}\left[C(1-A)+2 C^{\prime}\right]+h_{N}\right) / \bar{\lambda}_{11} & \bar{T}_{2}=h_{N} T_{2} / \bar{\lambda}_{11} & d_{2}=\delta \nu B / \bar{\lambda}_{11} \\
c_{3}=-\alpha \lambda_{11} C B / \bar{\lambda}_{11} & \bar{T}_{3}=h_{N} T_{3} / \bar{\lambda}_{11} & d_{3}=\delta\left[\bar{\lambda}_{10}-\nu_{10} A\right] / \bar{\lambda}_{11}
\end{array}
$$

Again, we have a Lyapunov function

$$
L=y_{1}^{2}+\frac{\bar{\lambda}_{11}}{\nu_{10} \bar{\lambda}_{01}}\left(y_{2}^{2}+y_{3}^{2}\right)
$$

and a trapping region defined by

$$
L \leq\left(\frac{\bar{\lambda}_{11}}{\nu_{10} \bar{\lambda}_{01}}\right)^{2} \frac{\|\bar{T}\|^{2}}{c_{1}^{2}}
$$

In contrast to equations (15), the divergence of the vector field of this reduced system can change sign, so that volume elements are not necessarily shrinking.

As explained in section (4.2), the amplitude of the nonlinear interaction depends on $y_{1}$ through $A$ and $B$. In figure (9) the dependence of the interaction coefficient in (32.1) is shown. This figure 


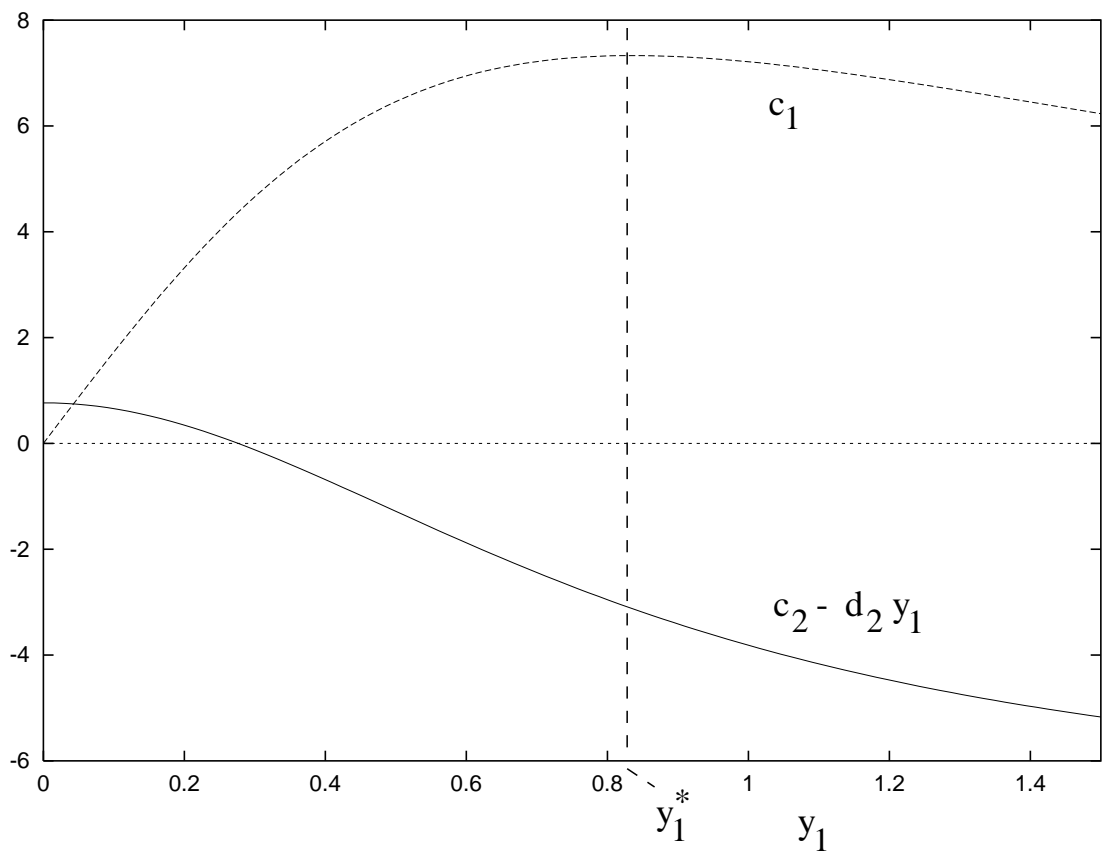

Figure 9: Dashed: the coefficient of interaction, $d_{1}$, between the jet stream pattern and the wave patterns. Solid: the effective damping coefficient of $y_{2,3}$. If we fix $y_{1}=y_{1}^{*}=\bar{C} / \kappa$ model (32) is equivalent to the Lorenz-84 model described in section (6)

illustrates the life cycle of the baroclinic waves. The linear stability of the Hadley circulation is determined by the effective damping coefficient of the wave components $y_{2,3}$, given by $c_{2}-d_{3} y_{1}$. If this number is positive, the waves are damped. If it is negative, a perturbation of the Hadley circulation will lead to growing waves. If $y_{1}$ is small, there is little energy transfer and the waves are damped. As $y_{1}$ is forced by the meridional temperature gradient, $\bar{T}_{1}$, it grows and the energy transfer increases. Beyond $y_{1} \approx 0.276$, the effective damping coefficient is negative, and baroclinic waves can grow. When $y_{1}=\bar{C} / \kappa \approx 0.83$, the phase shift, $\gamma$, is maximal and the waves are optimally baroclinic. They extract energy from the jet stream and $y_{1}$ decreases. Then the waves decay and become decreasingly baroclinic in the process.

In order to see, if system (32) behaves qualitatively the same as system (15), we study the bifurcation diagram. It is shown in figure (10). All the bifurcations displayed in diagram (2) are present here, too. Therefore, the qualitative behaviour of the reduced system (32) is the same as that of the full system (15). The essence of the extra degrees of freedom in the six dimensional model can be captured by the variable coefficients in the three dimensional model.

\section{Reduction to the Lorenz-84 model}

As a final simplification of our model, we fix the coefficients in equations (32). The choice

$$
A=A(\bar{C} / \kappa)=0 \quad B=B(\bar{C} / \kappa)=1
$$

maximizes the efficiency of the energy transfer between the baroclinic waves and the jet stream. The phase shift is fixed to $\gamma=\pi / 2$. Equivalently, we can reduce model (15) to the tangent space 


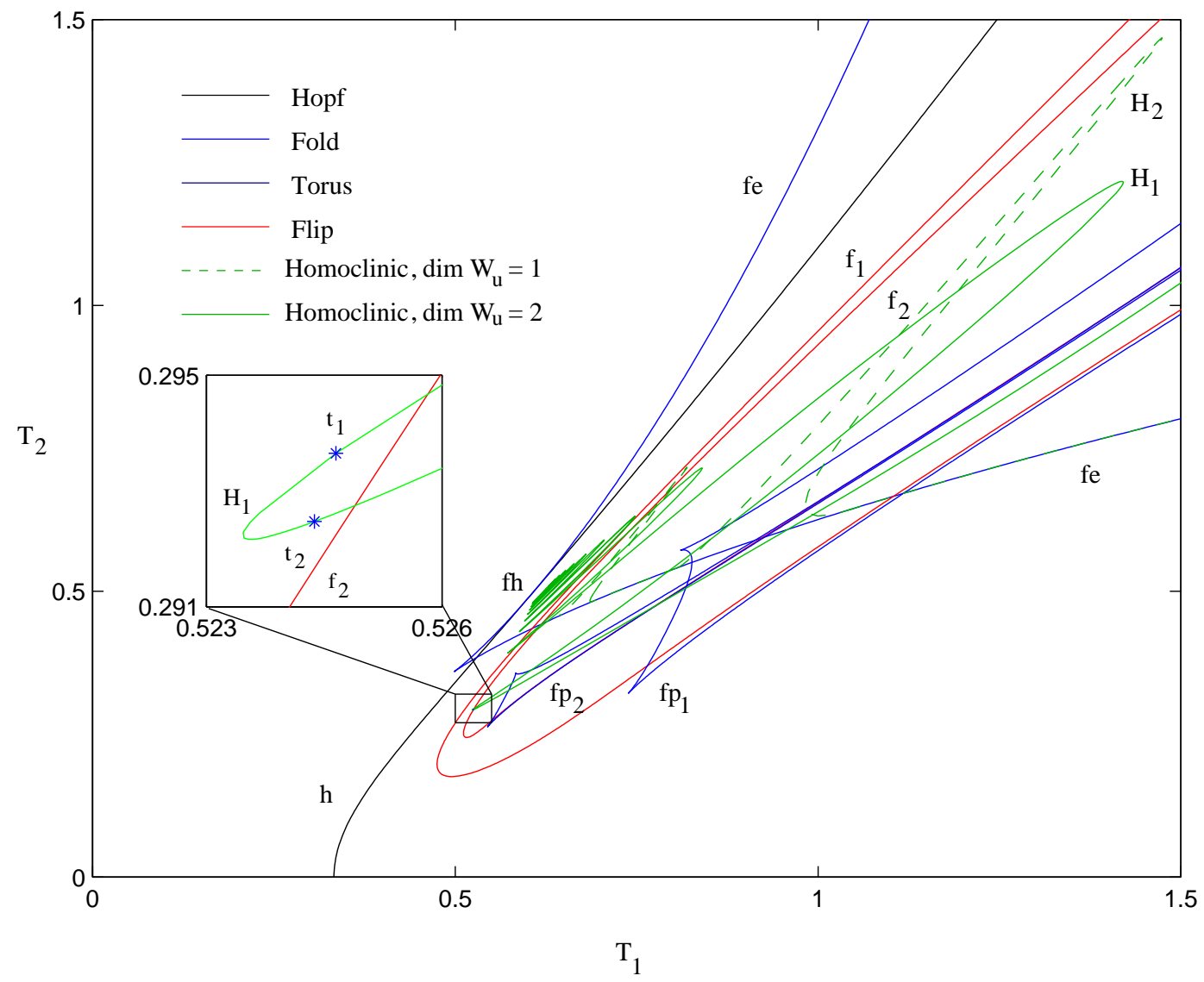

Figure 10: Bifurcation diagram of system (32). The enlargement shows the neutral saddle focus transitions $t_{1,2}$. Note the similarity to diagram (2)

$\mathrm{T}_{\left(\mathbf{x}^{*}, \mathbf{y}^{*}\right)} W_{0}$, where $\left(\mathbf{x}^{*}, \mathbf{y}^{*}\right)=(\bar{C} / \kappa, 0,0, \bar{C} / \kappa, 0,0)$. In other words, we linearise $\phi_{0}$ around the Hadley state.

We then scale $\mathbf{y}$ and $t$ according to

$$
\begin{aligned}
t & =\left[c_{2}+c_{3} \frac{d_{2}}{d_{3}}\right]^{-1} t^{\prime} & y_{1} & =\frac{1}{d_{2}}\left[c_{2}+c_{3} \frac{d_{2}}{d_{3}}\right] x-\frac{c_{3}}{d_{3}} \\
y_{2} & =\left[c_{2}+c_{3} \frac{d_{2}}{d_{3}}\right] \frac{1}{\sqrt{d_{1} d_{2}} y} y & y_{3} & =\left[c_{2}+c_{3} \frac{d_{2}}{d_{3}}\right] \frac{1}{\sqrt{d_{1} d_{2}}} z
\end{aligned}
$$

and find

$$
\begin{aligned}
& \dot{x}=-y^{2}-z^{2}-a x+a F \\
& \dot{y}=x y-b x z-y+G \\
& \dot{z}=b x y+x z-z
\end{aligned}
$$




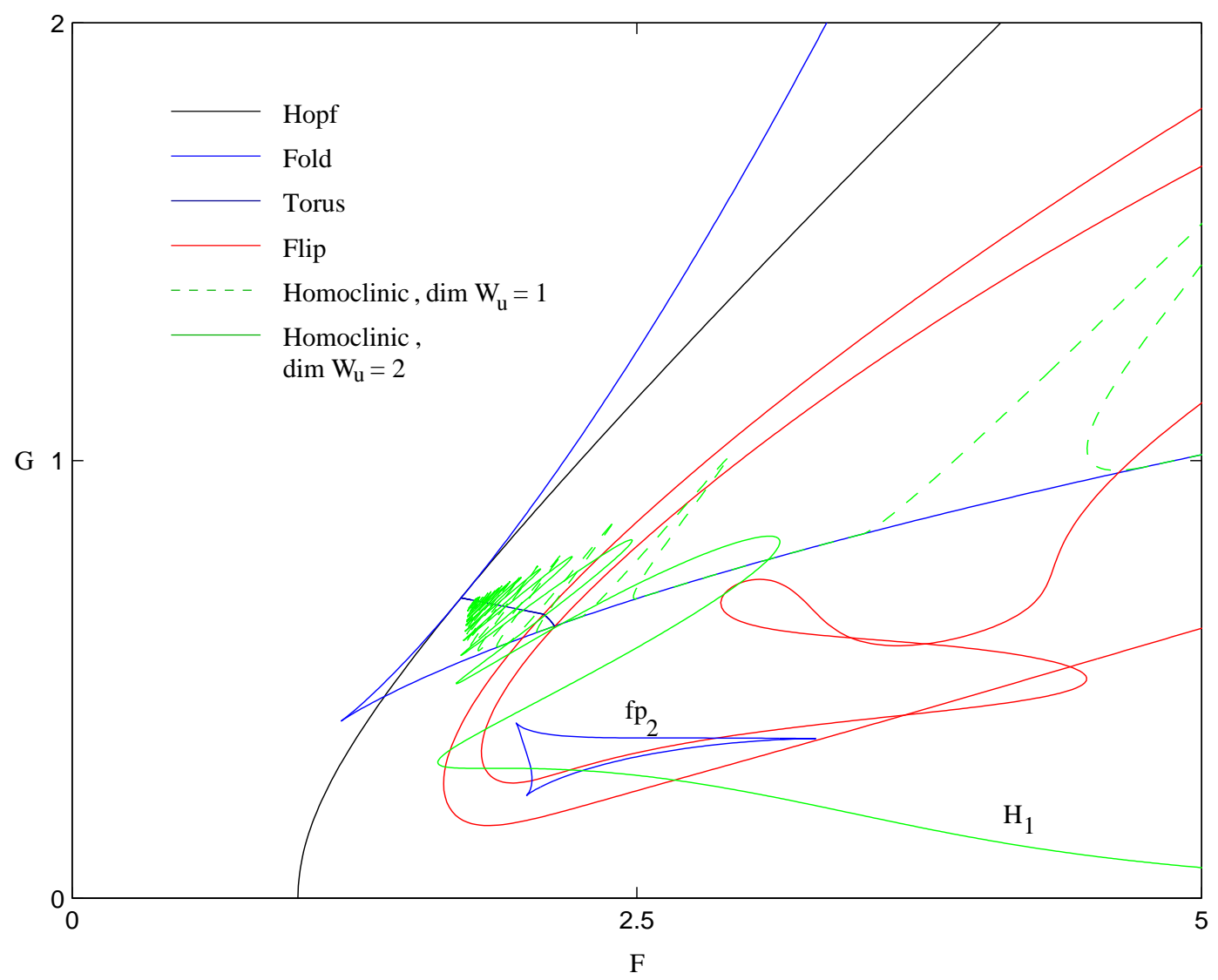

Figure 11: Bifurcation diagram of system (37), with parameters $a$ and $b$ as calculated from the physical parameters $C, C^{\prime}, h_{N}$ and $\alpha$.

which is the model introduced by Lorenz [1984]. For the parameters we find

$$
\begin{array}{rlrl}
F & =d_{2}\left[c_{2}+c_{3} \frac{d_{2}}{d_{3}}\right]^{-2}\left(\bar{T}_{1}+\frac{c_{1} c_{3}}{d_{3}}\right) & G & =\sqrt{d_{1} d_{2}}\left[c_{2}+c_{3} \frac{d_{2}}{d_{3}}\right]^{-2} \bar{T}_{2} \\
a & =c_{1}\left[c_{2}+c_{3} \frac{d_{2}}{d_{3}}\right]^{-1} & b & =\frac{d_{3}}{d_{2}}
\end{array}
$$

With the parameters as specified in section (4.1), we thus obtain

$$
a \approx 0.35 \quad b \approx 1.33
$$

in contrast to the traditional values $a=1 / 4$ and $b=4$. Diagram (11) has been obtained by setting the parameters according to (39). There still is a strong similarity to diagrams (2) and (10). However, the neutral saddle focus transitions have disappeared. Along both homoclinic bifurcation curves only a stable cycle is created. The accumulation of $1: 2$ resonance points is still there and, although homoclinic curve $\mathrm{H}_{1}$ moves farther away, the branch switching mechanism along $\mathrm{fp}_{2}$ works as described in section (4.1). 


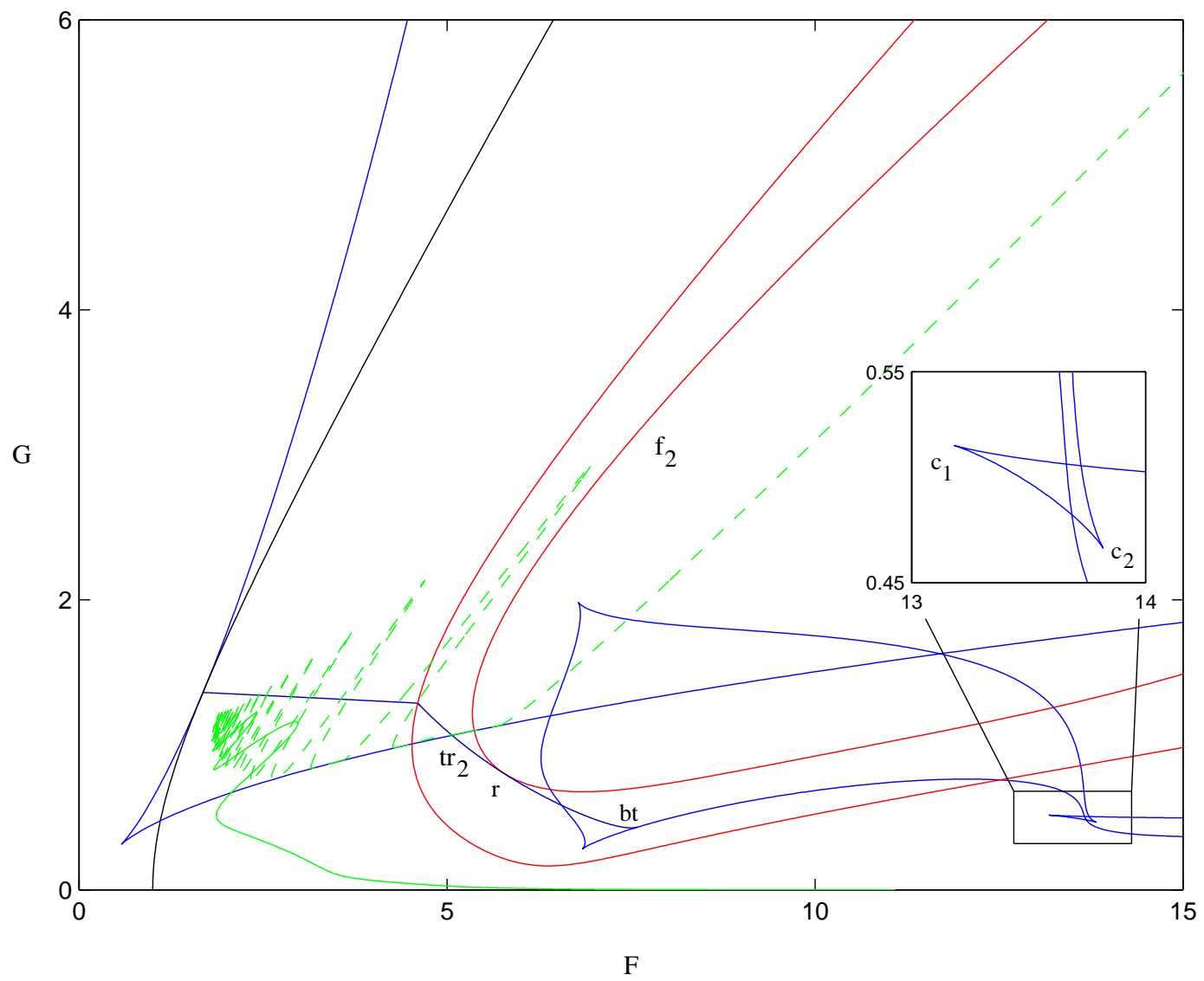

Figure 12: Bifurcation diagram of system (37), with $a=1 / 4$ and $b=3.24$. Torus bifurcation line $\operatorname{tr}_{2}$ and flip bifurcation line $f_{2}$ are tangent at point $r$. $\operatorname{tr}_{2}$ ends in a $1: 1$ resonance, or Bogdanov-Takens point, marked bt. The enlargement shows that extra cusps, $\mathrm{c}_{1,2}$, have developed on saddle node line $\mathrm{fp}_{2}$. Colour coding as in diagram (11).

\subsection{Continuation in $a$ and $b$}

Finally, we continue the bifurcations in diagram (11) in parameters $a$ and $b$ in order to establish the relation to the diagram at traditional parameter values, presented in Shilnikov et al. [1995]. Changing $a$ to its traditional value, $a=1 / 4$, does not change the bifurcation diagram qualitatively. When changing $b$ two changes are apparent. Shown in figure (12) is the diagram obtained for $a=1 / 4$ and $b=3.24$. The second torus bifurcation line, $\operatorname{tr}_{2}$, connecting two $1: 2$ resonance points in diagram (11), is now tangent to flip bifurcation line $\mathrm{f}_{2}$ at point $\mathrm{r}$ and connects to the fold line $\mathrm{fp}_{2}$. These two meet in a 1:1 resonance points, also called Bogdanov-Takens point.

Also, a pair of cusp points, marked $\mathrm{c}_{1,2}$, has developed on fold line $\mathrm{fp}_{2}$. These cusps denote creation and vanishing of successive wiggles on the branch of periodic solutions connected to homoclinic curve $\mathrm{H}_{1}$.

Figure (13) shows the bifurcation diagram at parameter values $a=1 / 4$ and $b=4$. This diagram was presented by Shilnikov et al. [1995]. The torus bifurcation line $\operatorname{tr}_{2}$ no longer connects to flip bifurcation line $\mathrm{f}_{2}$ and, consequently, has developed an angular degeneracy (Peckham et al. [1995) at point $\mathrm{D}$. Along curve $\operatorname{tr}_{2}$ the multipliers of the periodic orbit are given by $\exp ( \pm \mathrm{i} \phi)$, where $\phi$ is the phase angle. At point $\mathrm{D}$ it has a maximum given by $\phi \approx 0.8 \pi$. 


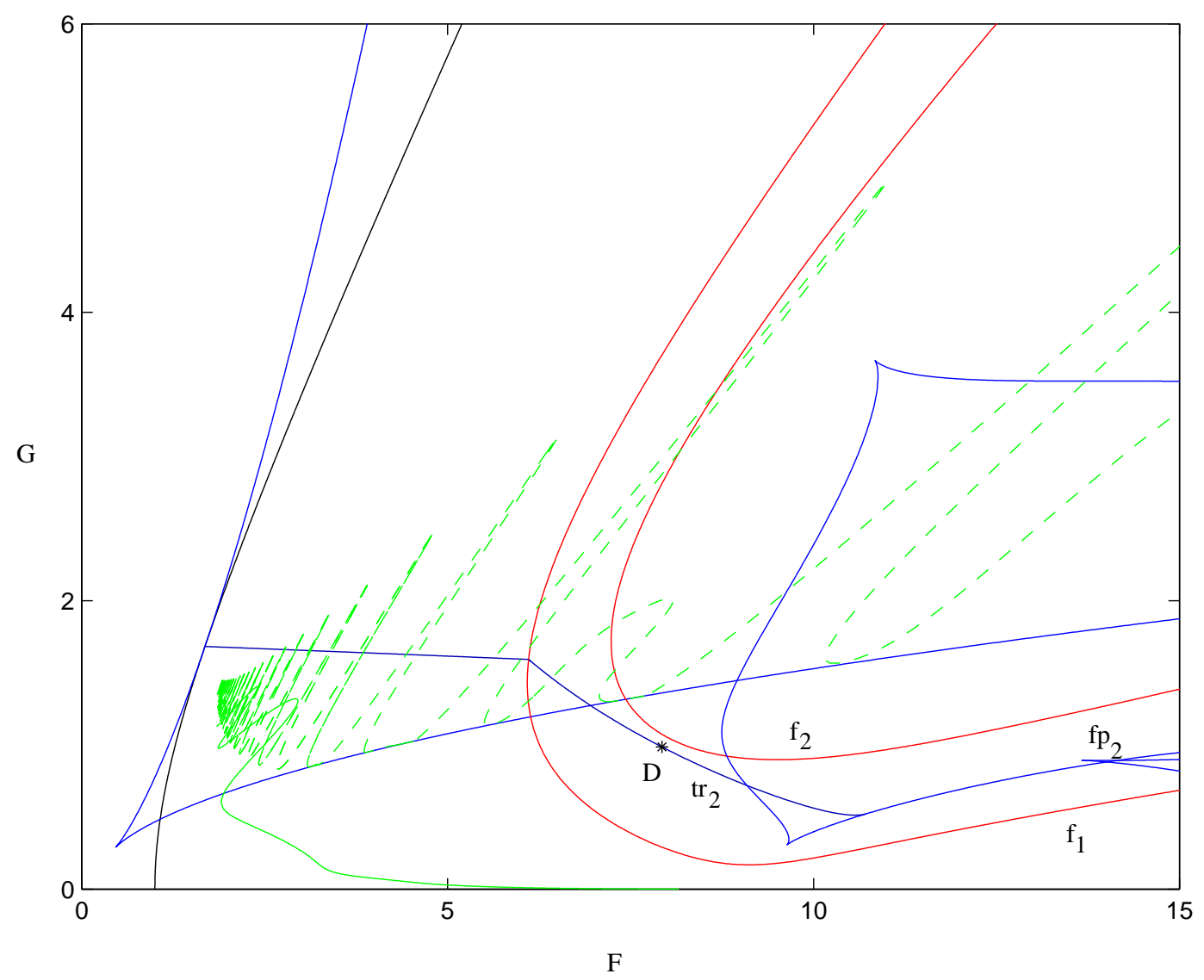

Figure 13: Bifurcation diagram of system (37), with $a=1 / 4$ and $b=4$, see also Shilnikov et al. [1995. Colour coding as in diagram (11). The angular degeneracy point on $\operatorname{tr}_{2}$ is marked $\mathrm{D}$.

If the forcing is set to $(F, G)=(8,1)$ the model is known to behave chaotically (e.g. Lorenz [1984]). An important, unanswered question is how this chaotic behaviour is brought about. With the derivation of the model and the bifurcation analysis in mind, we discuss four different possible routes to chaos in the following subsections.

\subsubsection{Period doubling cascades}

The flip bifurcation lines $f_{1,2}$ in diagram (13) still are the first of an infinite sequence. However, as explained in section (4.1), figure (5), a cross section with $F$ fixed does not show a period doubling cascade followed by an inverse cascade, due to branch switching along the cusped saddle node line $\mathrm{fp}_{2}$. The flip bifurcation lines $\mathrm{f}_{1,2, \ldots}$, extending beyond the limits of diagrams (11)-(13), are closed curves. As can be seen in diagram (11), these curves form unnested islands. In accordance with the claim to general applicability of the analysis presented in Wieczorek et al. [2001], the bifurcation diagram of the Lorenz- 84 model has much in common with that of their rate equation model. Chaotic attractors can be created and destroyed through different routes, including period doubling cascades, the Ruelle-Takens scenario and intermittency. 


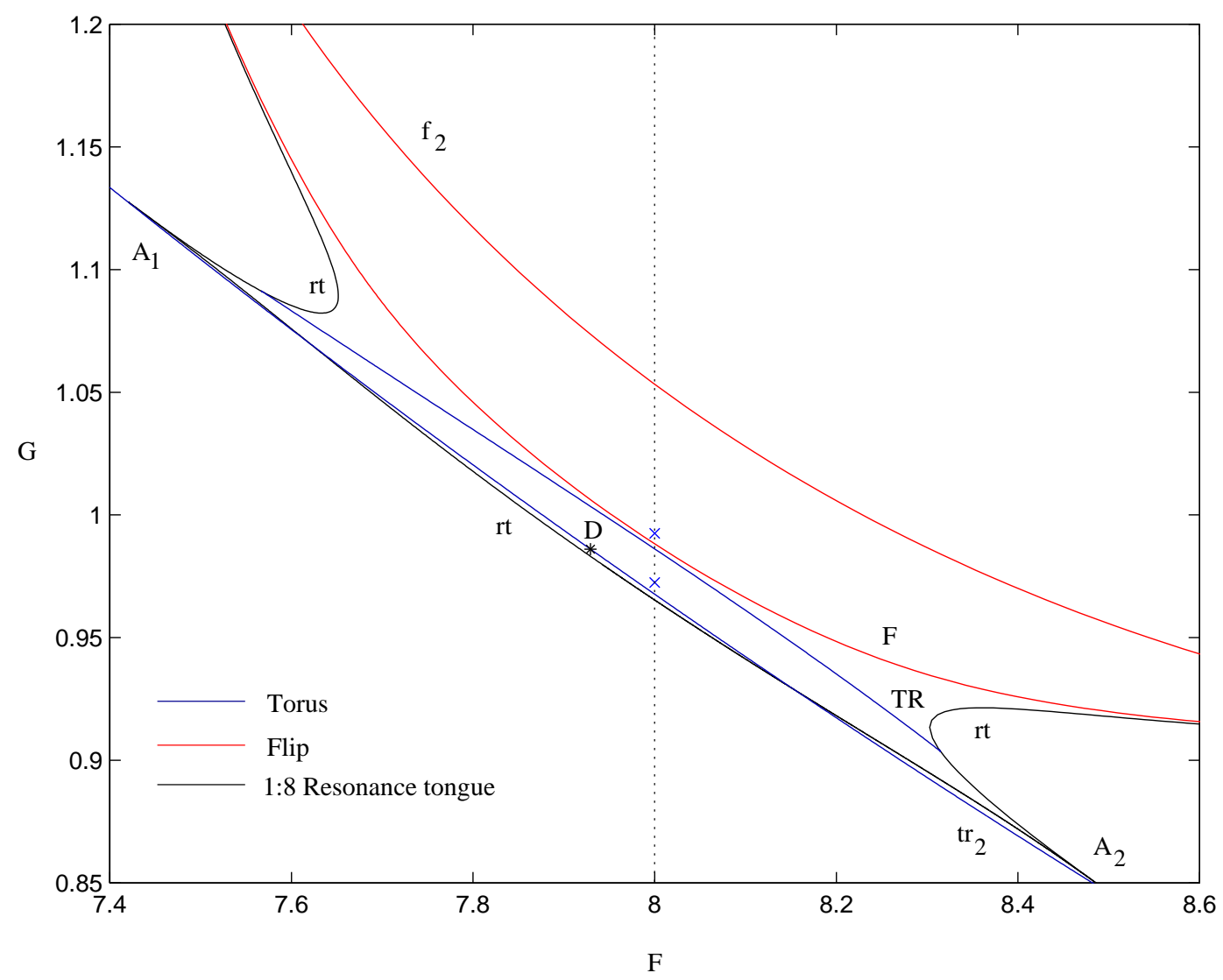

Figure 14: Detail of diagram 137. Added: boundaries of the $8: 1$ resonance tongue and a torus and flip bifurcation of the period 8 orbit. The crosses mark the parameter values of Poincaré sections in figure (15).

\subsubsection{Ruelle-Takens scenario}

Another possible route to chaos was proposed by Ruelle and Takens [1971]. In this scenario, an invariant torus is created first. Then a periodic obit appears on the torus, when crossing the boundary of an Arnold resonance tongue. If this periodic orbit bifurcates, a chaotic attractor can be created. This scenario can be observed in the Lorenz- 84 model. We will concentrate on the chaotic behaviour at parameter values $(F, G)=(8,1)$, close to the angular degeneracy point D.

If we fix $F=8$ and increase $G$ from below $\operatorname{tr}_{2}$, we find the attracting period two orbit first, then quasiperiodic behaviour in a small interval, then a period 8 orbit on the invariant torus. Figure (14) shows a detail of diagram (13), with the boundaries of the $8: 1$ resonance tongue and two subsequent bifurcations, a torus and a flip bifurcation, of the period 8 orbit.

The phase angle crosses the point $\phi=3 \pi / 4$ twice, at resonance points $\mathrm{A}_{1,2}$. One edge of the resonance tongue connects these points. It appears that the invariant torus itself goes through two subsequent fold bifurcations, so that the edge of the resonance tongue can cross $\operatorname{tr}_{2}$, and in a narrow band two stable tori coexist. This is shown in figure (15a). On the inner torus the dynamics is quasiperiodic, on the outer torus the period 8 orbits exist. If we further increase $G$, the period 8 orbit loses its stability in a torus bifurcation, directly followed by a flip bifurcation, as one of the multipliers crosses back into the unit circle through -1 . Beyond these bifurcations, a chaotic 

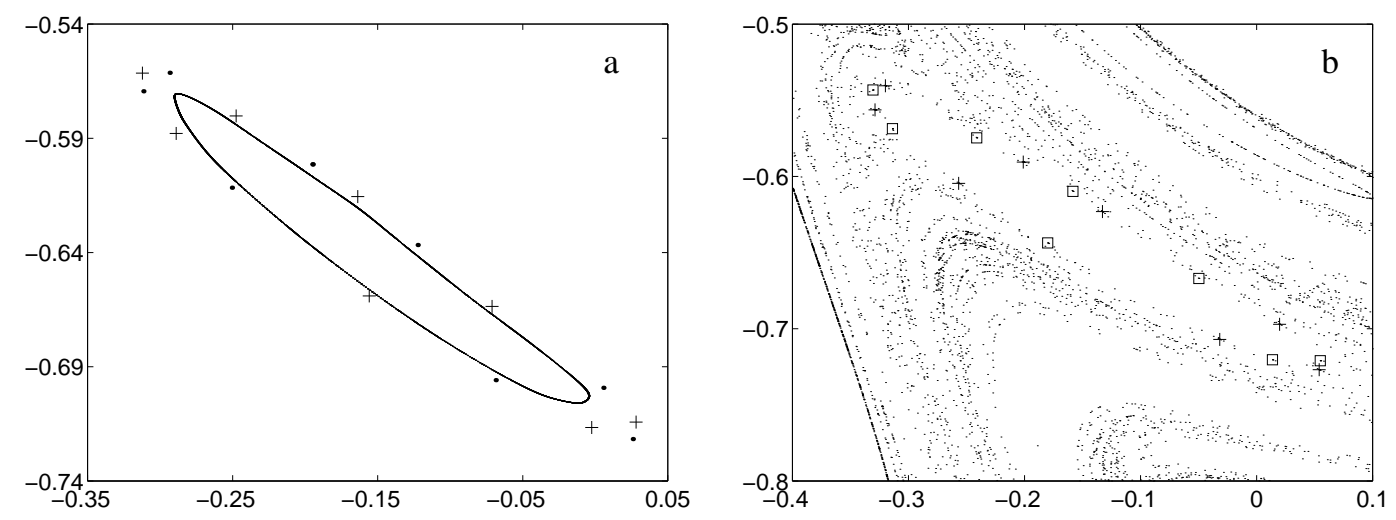

Figure 15: Poincaré sections of the Lorenz-84 model with $a=1 / 4$ and $b=4$. a: $(F, G)=(8,0.9725)$. Two coexisting invariant tori, one with the period 8 orbits, one with quasiperiodic dynamics. The thick dots denote the stable orbit, the crosses the saddle type orbit. b: $(F, G)=(8,0.99)$. Beyond the torus and flip bifurcations TR and F. Both orbits, marked with crosses and boxes are now of saddle type.

attractor appears. The corresponding Poincaré section is shown in figure (15) $\mathrm{b})$. The whole picture is more involved, as the tori shown in figure (15) a coexist with several tori with phase locked orbits of higher period.

Scenarios for the creation of a chaotic attractor through the bifurcation of a periodic orbit on on invariant torus are described in Broer et al. [1998]. The Ruelle-Takens scenario in the vicinity of an angular degeneracy was recently found in an electronic model by Algaba et al. [2001].

\subsubsection{Shil'nikov bifurcations}

The neutral saddle focus transitions, found in the six dimensional model, (15), and the approximate reduced model, (32), are not present in diagrams (11)-(13). Therefore, no Shil'nikov type chaos occurs in the Lorenz-84 model for these parameter values. We can, however, retrace the transition points for different values of $b$. As the continuation package HomCont (Doedel et al. [1986]) allows for three parameter continuation of codimension two points on homoclinic curves, it is possible to calculate a curve of neutral saddle focus transitions in the space of parameters $b, F$ and $G$. It turns out, that two transition points appear on curve $\mathrm{H}_{1}$ if $b<0.419$. Just like in diagram (10), on a small segment the saddle value becomes negative. Therefore, for $b<0.419$ Shil'nikov type chaos can be encountered in the Lorenz-84 model.

\subsubsection{Intermittency}

The last route to chaos described here is through intermittency. If we keep $F=8$ fixed and increase $G$, we find a periodic window around $G \approx 1.167$. At $G=1.16742$, the stable periodic orbit undergoes a saddle node bifurcation. Beyond this point, the behaviour is intermittent. In terms of Pomeau and Manneville [1980], this is type I intermittency. Two time series in the intermittent regime are shown in figure (16). In Wieczorek et al. [2001] the same type of intermittency is found and pictures of the stable and unstable manifolds of the saddle type orbit are shown. 

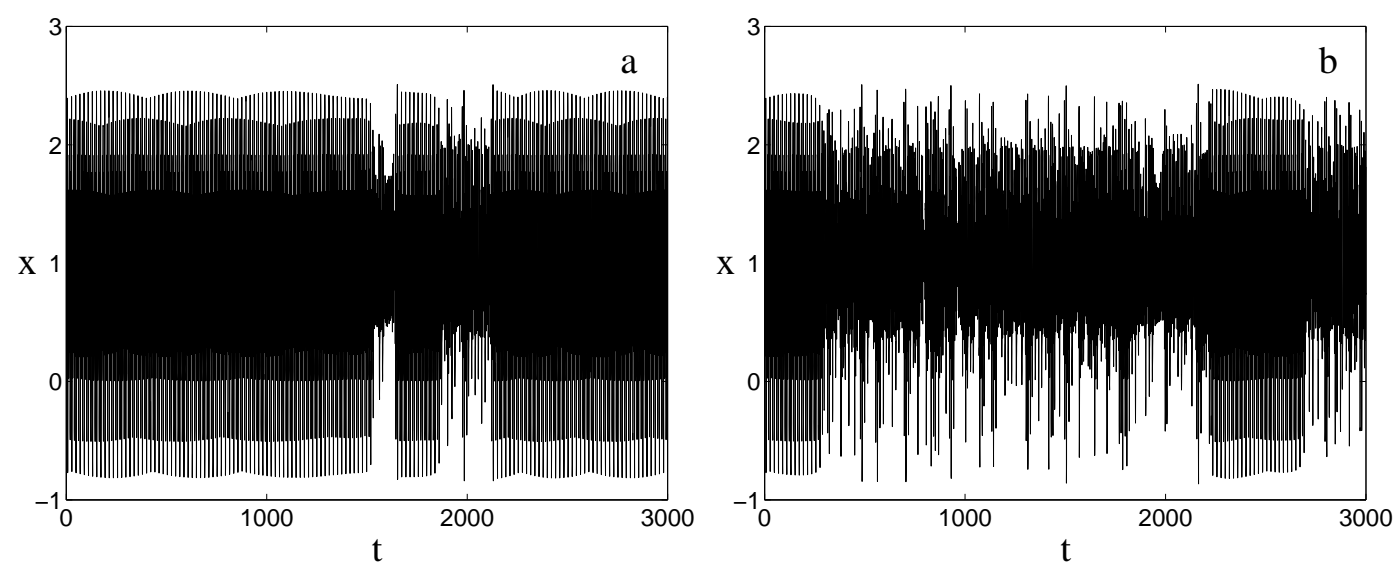

Figure 16: Intermittency in the Lorenz-84 model. a: $(F, G)=(8,1.16743)$. b: $(F, G)=(8,1.1675)$.

\section{Conclusion}

The starting point of the analysis in this paper is the truncation to six degrees of freedom of a QG two level model of atmospheric flow. Period doubling cascades and Shil'nikov bifurcations are identified as routes to chaos in the bifurcation diagram of this model. A measurement of the dimension of the chaotic attractor along a section of the bifurcation diagram reveals that it is less than three dimensional, hinting at the existence of a three dimensional invariant manifold which captures the dynamics.

In order to approximate this invariant manifold we have introduced a small parameter, $\epsilon$, into the equations. In the limit of $\epsilon \downarrow 0$ an exact solution was presented. This solution has a clear physical interpretation in terms of energy exchange between the zonally symmetric jet stream mode and the traveling waves. Numerical evidence for the existence of this manifold for finite $\epsilon$ was found implementing a variant of the graph transform algorithm of Broer et al. [1997].

We have shown that we can set $\epsilon=0$ without destroying the qualitative dynamics. The bifurcation diagram of the reduced model in this limit agrees well with the diagram of the six dimensional model. The invariant manifold at $\epsilon=0$ can be linearised around the Hadley state. If the six dimensional model is reduced to this linear approximation of the invariant manifold, the Lorenz- 84 model is found. The parameters of the Lorenz- 84 model can then be calculated from the physical parameters of the QG model. One of them comes out significantly different. We have compared the bifurcation diagram of the Lorenz- 84 model at physical parameters to that of the six dimensional model and to the diagram at traditional parameter values, first presented in Shilnikov et al. [1995].

Finally, we have discussed four possible routes to chaos in the Lorenz- 84 model. Period doubling cascades appear both in the six dimensional model and in the Lorenz- 84 model. Shil'nikov bifurcations are only found in the Lorenz- 84 model for parameter values far away from those considered here. A route to chaos not considered before in this model is the Ruelle-Takens scenario. We have presented evidence that the creation and destruction of invariant tori and the presence of resonance tongues lead to chaos in the Lorenz- 84 model. Finally, an intermittent transition has been presented. The overall picture of chaotic attractors being created and destroyed via different routes is reminiscent of the dynamics described in Wieczorek et al. [2001].

The link between a Galerkin truncation of a QG baroclinic model and the Lorenz-84 model justifies the use of the latter in conceptual studies of atmosphere and climate dynamics. It is remarkable how much of the bifurcation structure of the six dimensional truncation is preserved, 
notwithstanding rough approximations (namely setting $\epsilon=0$ and linearising the three dimensional invariant manifold). Probably, this is because the six dimensional model isolates one aspect of midlatitude, synoptic flow: the energy exchange between the jet stream and the baroclinic waves. When reducing to three degrees of freedom, and subsequently to the Lorenz-84 model, this process is modeled qualitatively correctly.

\section{Acknowledgments}

The author wishes to thank Ferdinand Verhulst, Theo Opsteegh, Ulrich Achatz, Yuri Kuznetsov and Renato Vitolo for useful discussions and proofreading. 


\section{References}

Achatz, U. \& Branstator, G. [1999] "A two-layer model with empirical linear corrections and reduced order for studies of internal climate variability," J. Atmos. Sci. 56, 3140-3160.

Algaba, A., Merino, M. \& Rodrígues-Luis, A. J. [2001] "Takens-bogdanov bifurcations of periodic orbits and arnold's tongues in a three dimensional electronic model," Internat. J. Bifur. Chaos $\mathbf{2}, 513-531$.

Broer, H. W., Osinga, H. M. \& Vegter, G. [1997] "Algorithms for computing normally hyperbolic invariant manifolds," Z. angew. Math. Phys. 48, 480-524.

Broer, H. W., Simó, C. \& Tatjer, J. C. [1998] "Towards global models near homoclinic tangencies of dissipative diffeomorphisms," Nonlinearity 11, 667-770.

Champneys, A. R. \& Kuznetsov, Yu. A. [1994] "Numerical detection and continuation of codimension-two homoclinic bifurcations," Internat. J. Bifur. Chaos 4, 785-822.

De Swart, H. E. [1988] "Low order spectral models of the atmopheric circulation: a survey," Acta Appl. Math. 11, 49-96.

Doedel, E., Champneys, A., Fairgrieve, T., Kuznetsov, Yu. A., Sandstede, B. \& Wang, X.J. [1986] AUTO9\%: Continuation and bifurcation software for ordinary differential equations (with HomCont) Computer Science, Concordia University, Montreal, Canada.

Foias, C., Jolly, M. S., Kevrekidis, I. G., Sell, G. R. \& Titi, E. S. [1988] "On the computation of inertial manifolds," Phys. Lett. A 131, 433-436.

Frisius, T. [1998] "A mechanism for the barotropic equilibration of baroclinic waves," J. Atmos. Sci. 55, 2918-2936.

Glendinning, P. \& Sparrow, C. [1984] "Local and global behavior near homoclinic orbits," J. Stat Phys. 35, 645-696.

González-Miranda, J. M. [1997] "Predictability in the lorenz low-order general atmospheric circulation model," Phys. Lett. A 233, 347-354.

Holton, J.R. [1992] Introduction to dynamic meteorology (Academic press).

Itoh, H. \& Kimoto, M. [1996] "Multiple attractors and chaotic itinerancy in a quasigeostrophic model with realistic topography: implications for weather regimes and low-frequency variability," J. Atmos. Sci. 53, 2217-2231.

Kuznetsov, Yu. A. [1998] Elements of applied bifurcation theory (Springer, New York).

Kuznetsov, Yu. A. [1999] "Numerical normalization techniques for all codim 2 bifurcations of equilibria in ode's," SIAM J. Numer. Anal. 36, 1104-1124.

Kuznetsov, Yu. A. \& Levitin, V. V. [1997] CONTENT: A multiplatform environment for analyzing dynamical systems Centrum voor Wiskunde en Informatica, Amsterdam.

Lorenz, E. N. [1960] "Energy and numerical weather prediction," Tellus 12(4), 364-373.

Lorenz, E. N. [1963] "Deterministic non-periodic flows," J. Atmos. Sci. 20, 130-141.

Lorenz, E.N. [1984] "Irregularity: a fundamental property of the atmosphere," Tellus 36A, 98-110. 
Peckham, B. B., Frouzakis, C. E. \& Kevrekidis, G. [1995] "Bananas and banana splits: a parametric degeneracy in the hopf bifurcation for maps," SIAM J. Math. Anal. 26, 190-217.

Peixoto, J. P. \& Oort, A. H. [1992] Physics of climate (Springer, New York).

Philips, N. A. [1954] "Energy transformations and meredional circulations associated with simple baroclinic waves in a two-level, quasi-geostrophic model," Tellus 6, 273-286.

Pielke, R. \& Zeng, X. [1994] "Long-term variability of climate," J. Atmos. Sci. 51, 155-159.

Pomeau, Y. \& Manneville, P. [1980] "Intermittent transition to turbulence in dissipative dynamical systems," Commun. Math. Phys. 74, 189-197.

Roebber, P.J. [1995] "Climate variability in a low-order coupled atmosphere-ocean model," Tellus 47A, 473-494.

Ruelle, D. \& Takens, F. [1971] "On the nature of turbulence," Commun. math. Phys. 20, 167-192.

Saltzman, B. [1962] "Finite amplitude free convection as an initial value problem," J. Atmos. Sci. 19, 329 .

Shilnikov, A., Nicolis, G. \& Nicolis, C. [1995] "Bifurcation and predictability analysis of a low-order atmospheric circulation model," Internat. J. Bifur. Chaos 5(6), 1701-1711.

Veen, L.van, Opsteegh, T. \& Verhulst, F. [2001] "Active and passive ocean regimes in a low-order climate model," Tellus 53A, 616-627.

Wieczorek, S., Krauskopf, B. \& Lenstra, D. [2001] "Unnested islands of period doublings in an injected semiconductor laser," Phys. Rev. E 64, 056204.

Wiin-Nielsen, A. [1992] "Comparisons of low-order atmospheric dynamical systems," Atmosfera 5, $135-155$ 